\title{
Mapping coalescence of micron-sized drops and bubbles
}

\author{
Joseph D. Berry ${ }^{1} \&$ Raymond R. Dagastine*
}

Department of Chemical and Biomolecular Engineering, University of Melbourne, Parkville VIC 3010, Australia.

Particulate Fluids Processing Centre, University of Melbourne, Parkville VIC 3010, Australia

\begin{abstract}
Emulsion formulation, solvent extraction and multiphase microfluidics are all examples of processes that require precise control of drop or bubble collision stability. We use a previously validated numerical model to map the exact conditions under which micron-sized drops or bubbles undergo coalescence in the presence of colloidal forces and hydrodynamic effects relevant to Brownian motion and low Reynolds number flows. We demonstrate that detailed understanding of how the equilibrium surface forces vary with film thickness can be applied to make accurate predictions of the outcome of a drop or bubble collision when hydrodynamic effects are negligible. In addition, we illuminate the parameter space (i.e. interaction velocity, drop deformation, interfacial tension, etc.) at which hydrodynamic effects can stabilise collisions that are unstable at equilibrium. Further, we determine conditions for which drop or bubble collisions become unstable upon separation, caused by negative hydrodynamic pressure in the film. Lastly, we show that scaling analyses are not applicable for constant force collisions where the approach timescale is comparable to the coalescence timescale, and demonstrate that initial conditions under these circumstances cannot be ignored.
\end{abstract}

\footnotetext{
${ }^{*}$ Corresponding author. Email address: rrd@unimelb.edu.au

${ }^{1}$ Email address: joe.d.berry@gmail.com
} 


\section{Introduction}

Interactions of micron-sized bubbles and droplets separated by distances from nanometres to microns are important in many applications, including food processing, cosmetics, mineral flotation and microfluidics. Drops and bubbles of this size are continually in relative motion, induced by processes including Brownian dynamics, flows generated in microfluidics, and gravity. As a consequence of this relative motion, bubbles or drops in emulsions or foams are continually colliding and separating under force fields influenced by i) the equilibrium interaction forces that arise from the surface chemistry of drops and bubbles, ii) the dynamic interaction forces due to the motion of the fluid and interface, and iii) the transport of neutral and charged species on, to and around the bubble and drop surface. The relative velocity of these interactions span many orders of magnitude, from Brownian-induced motion of $O(1 \mu \mathrm{m} / \mathrm{s})$ [1] to drop collisions in microfluidics of $O(10 \mathrm{~mm} / \mathrm{s})$ or higher[2] [3]. If the combination of these equilibrium and dynamic interactions are unstable, then coalescence will occur. Yet, the interplay between the equilibrium interactions, dynamic processes and the deformation of the drop or bubble interface can make precise understanding of the conditions under which coalescence occurs difficult. Importantly, this understanding is vital in a range of applications including the prediction of emulsion stability or shelf life in formulated products, drop or bubble coalescence phenomena in processing equipment such as mixer-settlers, and in assessing the performance of high-throughput multiphase microfluidic devices. For example, in these devices, droplets are generated at rates of up to thousands of drops per second $[4,5]$, and are required to transport reagents or biological material in discrete packages. In such devices, it is critical that interactions between drops do not lead to coalescence. In other processes, such as liquid-liquid separation, it is desirable to enhance and accelerate drop coalescence[6].

Equilibrium interactions, whereby the collision speeds are too low for hydrodynamic pressure to play a significant role, have been extensively characterised for deformable drops and bubbles. Building on methods for flat liquid-liquid or liquid-gas interfaces, such as thin film balance methods to measure pressure and thickness[7-9], first pioneered by Sheluko[10] and Mysels[11], and later developed by Wasan and coworkers $[12,13]$, there has been a steady evolution of methods to probe the equilibrium surface forces between deformable interfaces with curvature for drop and bubble radii near the capillary length or smaller. This includes the liquid surface forces apparatus[14], the work from the Horn group using a modified surface force apparatus using a mercury $[15,16]$ or gas[17] filled capillary and a rigid flat surface, and the extension of atomic force microscopy (AFM) to probe the interactions between, first, a rigid particle and a single bubble[18-20] or drop[21-24], and secondly, to measure the interactions of drop[25-27] or bubble[28] pairs. There have also been more recent developments, including the integration of interferometry with AFM to probe a single bubble with a flat surface[29], larger length-scale systems that utilise bimorph cantilevers and a single drop or bubble in the integrated thin film drainage apparatus (ITFDA)[30], and cantilevered capillaries to examine the interaction of two drops [31]. The range of surface forces examined in these experiments include Derjaguin-Landau-Verwey-Overbeek (DLVO) forces (the addition of electrical double layer and van der Waals) [25, 32, 33], repulsive van der 
Waals forces [34, 35], steric [36, 37], structural [36, 38, 39], depletion [36, 40], protein interactions [36, 41] and hydrophobic forces [42]. Common surface forces and their corresponding pressure and energy definitions are given in Tables S1 \& S2 in the Supplementary Information (SI). Equilibrium interactions can lead to repulsive or "stable" drop/bubble interactions or "unstable" interactions ending in coalescence [43].

At higher velocities, $O(10 \mu \mathrm{m} / \mathrm{s})$ and above, hydrodynamic drainage plays an important role in drop/bubble collisions. The AFM was easily extended to probe hydrodynamic drainage for a particle and drop first by Aston and Berg [44] and then for drops [26] and bubble [28] pairs. In addition, a number of the methods mentioned above, including the work by Horn[15-17, 45], classical drainage studies of drops immobilized in larger capillaries[46], the ITFDA and the cantilevered capillary measurement, have all examined a wide range of hydrodynamic drainage behaviour. For example, stable interactions have been observed with both stable and unstable equilibrium forces[28, 42]. Coalescence has also been observed for drops and bubbles in the above methods whilst on approach[47] and whilst separating[28], where coalescence whilst separating was first observed in four roll mill measurements[48] and then micro-fluidic devices[49, 50]. In addition, coalescence has been observed in drops and bubbles undergoing cyclic accelerating approach and withdrawal to mimic drop collisions in microfluidic devices [51], and whilst being held together at constant applied force to mimic buoyant collisions [52]. In dynamic collisions the viscosity of the continuous phase becomes an important parameter[53, 54]. Other important quantities that can affect dynamic drop and bubble coalescence are the presence of free charge, and in some practical applications the application of external electric fields [55-58].

The impact of experimental measurements has come to fruition though efforts to develop quantitative models to account for the presence of surface forces, deformation and hydrodynamic drainage (in a creeping flow, $\mathrm{Re}<<1$ regime) in order to properly analyse these data. In particular, the deformable nature of the system often adds complication to the measurements and analysis, particularly when the length scales of the deformation are not accessible with methods such as interferometry. In the area of AFM measurements with deformable interfaces, the analysis for equilibrium interactions were pioneered by several groups, first for a particle and single drop or bubble[32, 59, 60], then for dynamic interactions for a single drop[44], and then bubble and drop pairs[1]. The application and validation of these models to a range of experimental systems using AFM can be found in the review by Tabor et al.[43]. The summary of the development of these models can be found in the extensive review by Chan and co-workers[61]. The Stokes-YoungReynolds-Laplace (SYRL) numerical model, initially developed and validated for AFM measurements, has been extended to analyse the dynamic force measurements using the ITFDA [62] and several studies focused on the modified surface forces apparatus from the Horn group[63, 64]. In addition, the more recent work by Zeng using AFM to probe the interactions of a single drop or bubble with a flat plate using interferometry[29] was well described by the same model, first validated on similar measurements without interferometry[34, 65].

These observations, in combination with theory, have helped to shed light on the role of equilibrium interaction forces, interfacial deformation, drop/bubble size, 
interfacial/surface tension, viscosity, and collision velocity on the stability of interactions, and how these parameters affect the mode of coalescence (i.e. during approach, retract, or dwell) for unstable interactions. Thus, the observations in the literature and a comprehensively validated SRYL numerical model for both AFM measurements and force measurements at larger drop and bubble length scales suggest that is may be useful to apply this knowledge in a larger context.

The schematic in Figure 1 suggests juxtaposition of the AFM measurement to other types of measurements and uses of drops and bubbles (i.e. emulsion, foams and micro-fluidics) in terms of relative drop and bubble size. Emulsions or foams are commonly characterised by sub-micron radii (in the case of emulsions) to $10 \mathrm{~s}$ of microns in radii or larger in the case of some foams. AFM measurements have accessed drops as small as 5 microns in radius[66] (although there is little deformation in those instances) to drops and bubbles with radii up to several hundred microns. The micro-fluidic device, showing drop coalescence, denotes that microfluidic drops and bubbles can have radii commensurate with an AFM measurement or larger. The two drops fixed on capillaries represent the larger drop and bubble radii found in the ITFDA and cantilevered capillary studies as well as more classical capillary drainage studies, where the drop rise scenario is representative of traditional drop coalescence studies. Thus, in this study we attempt to move forward from AFM measurements on individual systems to decant aspects of this understanding to more general questions, mapping the precise conditions whereby drops and bubbles coalesce. Using the SRYL numerical model we systematically probe the multidimensional parameter space, in order to determine the conditions required for both stable and unstable interactions, and the mechanisms responsible for coalescence of micron-sized bubbles or drops with direct relevance to the scenarios shown in Figure 1.

\section{Method}

Theory

The dynamics of both bubble and drop collisions are controlled by the relationship between surface forces, fluid dynamics, and surface deformation. The stability of a drop or a bubble collision is governed by the dynamic drainage in the liquid film separating them. If the film between the two interacting drops or bubbles drains completely then coalescence will result, and the interaction is said to be unstable. Conversely, if the film does not have time to drain before the drops or bubbles move apart, then coalescence will not occur, and the interaction is defined as stable.

The SRYL model is well described in the literature[1, 61, 67], but due to the focus of this work on using this model, some of the key points are summarized here. The rate at which the film between two interacting, deformable drops or bubbles drains is governed by the equation:

$$
\frac{\partial h}{\partial t}=\frac{1}{12 \mu r} \frac{\partial}{\partial r}\left(r h^{3} \frac{\partial p}{\partial r}\right)
$$

where $h$ is the film thickness, $\mu$ is the viscosity of the liquid composing the film, $p$ is the hydrodynamic pressure, and $r$ is the radial coordinate. 
The normal stress balance for the deformation of drops or bubbles due to the disjoining pressure $\Pi$ and hydrodynamic pressure $p$ is given by the Young-Laplace equation with the added term for $p$ :

$$
\frac{\sigma}{2 r} \frac{\partial}{\partial r}\left(r \frac{\partial h}{\partial r}\right)=\frac{2 \gamma}{R}-p-\Pi \text {. }
$$

Here $\gamma$ is the interfacial tension and $R=2 R_{1} R_{2} /\left(R_{1}+R_{2}\right)$ is the harmonic mean of the two drop/bubble radii $R_{1}$ and $R_{2}$.

Together, Equations (1) \& (2) represent the SRYL model, which has been comprehensively validated for the AFM setup where a drop or bubble pinned to a cantilever is driven towards a drop or bubble resting on the substrate. The appropriate boundary conditions for this particular scenario are given at $\mathrm{r}=0$ as $\partial h(r, t) /\left.\partial r\right|_{r=0, t}=0$ and $\partial P /\left.\partial r\right|_{r=0, t}=0$. At $r_{\text {max. }}$, where the inner numerical solution is matched to the outer analytical solution of the drop or bubble profile, the additional boundary conditions are

$$
\begin{gathered}
\left.\frac{\partial P(r, t)}{\partial r}\right|_{r=r_{\text {max }, t} t}=-\frac{4 P\left(r=r_{\text {max. }}, t\right)}{r^{4}} \\
\left.\frac{\partial h(r, t)}{\partial t}\right|_{r=r_{\text {max }, t}}=-\frac{d \Delta X(t)}{d t}+\frac{1}{K} \frac{d F(t)}{d t} \\
-\frac{1}{2 \pi \gamma} \frac{d F(t)}{d t}\left[\log \left(\frac{R_{2}}{R_{1}}\right)+B\left(\theta_{1}\right)-B\left(\theta_{2}\right)\right] .
\end{gathered}
$$

Here $\Delta X$ is the drive position, $\mathrm{K}$ is the cantilever spring stiffness, $F(t)$ is the total interaction force, $\theta_{1}$ and $\theta_{2}$ are the contact angles of the drops on the cantilever and substrate respectively, and $B\left(\theta_{i}\right)$ is defined as

$$
B\left(\theta_{i}\right)=1+\frac{1}{2} \log \left(\frac{1+\cos \theta_{i}}{1-\cos \theta_{i}}\right)
$$

under the assumption that the contact lines of the drops and bubbles are pinned. In this study we remove the dependence upon the cantilever stiffness by making $\mathrm{K}$ very large, and consequently the second term on the RHS of Equation 4 is zero. The interaction force $F(t)$ is calculated from the pressure using

$$
F(t)=2 \pi \int_{0}^{\infty}(p+\Pi) r d r
$$

The SRYL model requires one initial condition, which is defined as $h(r, t=0)=$ $h_{0}+r^{2} / R$, where $h_{0}$ is the initial separation between the two bodies at $r=0$ such that the separation is sufficient to assume that neither body is deformed at $h_{0}$.

The governing Equations 1 and 2, together with the boundary conditions and the initial condition above, solved for a constant velocity approach-retract drive and different values of drive velocity $U$, initial separation $h_{0}$, and interfacial tension. It is assumed that the oil-water or air-water interfaces are immobile[25, 28, 61]. The value of $r_{\max }$ is chosen to be large enough such that its size has negligible effect on the calculations, where a more detailed discussion of the choice of $r_{\max }$ can be found in Manica et al. [67]. 
The SRYL model was built on the model to describe the equilibrium interactions between two drop or bubble pairs[60,61]. Thus, the SRYL model reduces to the Chan-Dagastine-White model for equilibrium interactions and is well summarised in the literature. In the interest of brevity, we will not repeat the expressions here, but they are nicely presented in the review paper by Tabor et al.[43].

\section{Model Systems}

In the models outlined above, bubbles and drops are uniquely defined through specification of interfacial tension, surface charge/potential, and surface forces. The key differences between bubbles and drops are interfacial tension ranges (with or without surfactant), which are generally higher for bubbles (i.e. air/water interfaces), and the size of the van der Waals force, which is almost an order of magnitude higher for a bubble than for a drop. In this study, we examine equilibrium and dynamic interactions of both bubble and drop pairs. We initially focus on systems exhibiting DLVO forces, which have are prevalent in nature and have been well characterised in the literature for bubbles [28, 68] and drops[25, 33, 35]. We also consider drops exhibiting (repulsive) steric forces [37] and also drops with (attractive) depletion forces[36, 40].

\section{Discussion and Results}

\section{Equilibrium Interactions}

Coalescence of drops and bubbles under equilibrium conditions (where hydrodynamic effects are minimal) is governed by the thermodynamic driving force to minimise the interfacial area of the two phases. Surface forces embody this basic thermodynamic principle when determining the stability of drop and bubble interactions. Thus, the starting point for determination of coalescence behaviour is to examine the coalescence behaviour as a function of equilibrium surface forces between deformable drops and bubbles as well as interfacial tension and drop or bubble radii.

In order to map the equilibrium coalescence behaviour of a drop pair in the presence of a DLVO force, we use the disjoining pressure between two flat tetradecane interfaces with a surface potential of $-17 \mathrm{mV}$ separated by a binary electrolyte solution with an ionic strength of $1 \mathrm{mM}$ as shown in Figure 2a. The form of the EDL pressure used in this calculation, assuming a weak overlap interaction, is given in Table S2 in the SI. The retarded van der Waals force was calculated using Lifshitz theory, where the dielectric spectra required were taken from Dagastine, et al. [69] for water and Parsegian and Weiss [70] for the tetradecane.

To elucidate the effect of interfacial tension, the equilibrium interactions between drops of radius $\mathrm{R}=40 \mu \mathrm{m}$ and different interfacial tensions interacting under the influence of this disjoining pressure, calculated using the Chan-Dagastine-White model [60], are shown in Figure 2b). For low interfacial tensions $(\gamma \leq 30 \mathrm{mN} / \mathrm{m})$, the interaction is repulsive with a stable film separating the two drops and no coalescence occurs. For higher interfacial tensions $(\gamma \geq 40 \mathrm{mN} / \mathrm{m})$, the interaction becomes unstable and coalescence occurs. The arrows in Figure $2 b$ denote the point at which these unstable interactions coalescence. In Figure $2 \mathrm{c}$, we have extracted the minimum separation in the film prior to coalescence or the minimum separation of the 
stable film as a function of interfacial tension where there is a transition from stable film to coalescence is at approximately $\gamma \sim 38 \mathrm{mN} / \mathrm{m}$.

The above example demonstrates that there is a critical interfacial tension where a system that has an attractive disjoining pressure, which can lead to coalescence, can also results in a stable interaction between drops due to the interfacial deformation of the system. How this stable film evolves is shown the case of a $30 \mathrm{mN} / \mathrm{m}$ interfacial tension in the subset of Figure 2c, where the interfacial separation profiles between the two drops correspond to the $x$ symbols on the $30 \mathrm{mN} / \mathrm{m}$ force curve. It is clear that the film approaches a minimum film separation, $h_{\text {stable }}$, at the axial $(\mathrm{r}=0)$ centre of film. As the film flattens, the minimum separation stops changing even as $\Delta X$ decreases and the force continues to increase, but this is due to the larger interaction area between as the two drops continue to deform. As the film begins to flatten, the derivative terms describing the curvature of the film near the axial centre begin to vanish in the Young-Laplace equation (Equation 2), thus we can simplify this equation at the centre of the drops $(\mathrm{r}=0)$ to

$$
\frac{2 \gamma}{R} \approx \Pi\left(h_{\text {stable }}\right)
$$

This relationship gives a criterion for the stability of equilibrium interactions. If the Laplace pressure term $2 \gamma / R$ is greater than the disjoining pressure for all separation distances $h$, then the interaction will be unstable and the drops will coalesce, assuming that the drops are already in close enough proximity to cause some amount of interfacial deformation. We can use Equation 7 to also predict the minimum film separation, $h_{\text {stable }}$.

Equation 7 is an asymptotic solution, and is valid at the centre of the drop $(r=0)$, however it neglects the significant curvature present at the edge of the interaction. Despite this approximation, it is adept at predicting the stability of equilibrium drop interactions. To demonstrate this, we plot the Laplace pressure for drops of radius $\mathrm{R}=$ $40 \mu \mathrm{m}$ and varying interfacial tensions with the disjoining pressure in Figure 2a. For interfacial tensions $\gamma \leq 35 \mathrm{mN} / \mathrm{m}$, the Laplace pressure is balanced by the disjoining pressure for all values of separation $h$, indicating that the film will cease thinning once this separation is reached, rendering the interaction stable. However, when the interfacial tension is increased to $\gamma \geq 40 \mathrm{mN} / \mathrm{m}$ the disjoining pressure is not high enough at any separation to balance the Laplace pressure of the drop, and the interaction is unstable. This prediction agrees extremely well with the results of the Chan-Dagastine-White model, shown in Figure $2 b$ and $2 c$, which accounts for the effect of deformation over the entire interaction area. Because the stability for criterion involves the Laplace pressure, the drop radius also plays a role in the stability of equilibrium interactions. For fixed interfacial tension, an increase in drop radius gives a decrease in the Laplace pressure, leading to stable interactions if the drop radius is increased sufficiently.

As a stability criterion, this relation can be generalised to any disjoining pressure versus separation model, not just the DLVO force used in Figure 2. This approach is not as general as explicitly deriving the effect of deformation on a stability ratio, but may offer more utility with much less complexity. An earlier study has attempted to account for deformation in stability ratio, but used truncated spheres instead of 
deformed surfaces [71]. Based on the interface profiles we can see this is not appropriate to describe the interaction of these interfaces, but accounting for complete interfacial profiles is difficult to incorporate into stability ratio theory.

\section{Dynamic Interactions}

When the velocity of the drop or bubble interaction is significant, the hydrodynamic pressure within the film is no longer negligible. The dynamic interactions of two air bubbles in water in the presence of a DLVO force are characterised in Figure 3. The EDL contribution is calculated from an implicit numerical solution of the PoissonBolzmann equation, and the non-retarded $\mathrm{vdW}$ contribution is calculated using the form in Table S2 in the SI. A bubble of radius $60 \mu \mathrm{m}$ is driven at speed $U$ towards a bubble of $45 \mu \mathrm{m}$ over a distance $X=20 \mu \mathrm{m}$, and then instantaneously retracted at the same speed $U$. A large number of numerical simulations were performed with different drive velocities $U(1-250 \mu \mathrm{m})$ and initial separations $h_{0}(8-20 \mu \mathrm{m})$, where the outcome of each interaction was classified into one of three categories: coalescence on approach, coalescence on retract, and no coalescence. The first two categories represent unstable interactions, and the last category represents a stable interaction. Instead of plotting the results in terms of initial separation $h_{0}$, we choose to use the quantity $X-h_{0}$, where $X$ is the total drive distance. This quantity represents the amount of "overlap" that the drops would experience if they were rigid bodies: for example, an overlap of $2 \mu \mathrm{m}$ means that the drops have been driven together $2 \mu \mathrm{m}$ past their initial separation. The overlap quantity can be thought of as a measure of the amount of deformation experienced by the drops, or alternatively a measure of the extent of the collision. In this study, we plot our stability maps in terms of drive velocity and overlap (or deformation). In practice, the interaction velocity and amount of deformation are interrelated, but the AFM (and hence model) setup allows us to decouple the two to isolate the effect of each.

For the bubble pair shown in Figure 3a, the air-water interface is assumed to be at a low surfactant coverage, and the interfacial tension is consequently $\gamma=70 \mathrm{mN} / \mathrm{m}$. We observe three different outcomes for this parameter space. First, for low interaction velocities, the film completely drains on the approach stage and coalescence occurs. The second outcome occurs for moderate interaction speeds, where the film does not completely drain before the approach ends. However, the film continues to drain during the retract stage and coalescence still occurs, despite the fact that the bubbles are moving apart. This behaviour is consistent with observations of drop coalescence during separation in microfluidic devices [49], glancing collisions in a four-roll mill [48] and bubble coalescence on retract in an AFM [28]. Thirdly, for very large interaction velocities, the film does not have sufficient time to drain over both the approach and retract stages, and no coalescence occurs. Thus, even though the surface forces are unstable at equilibrium, hydrodynamic effects stabilise these interactions at high velocities. Thus, hydrodynamic effects are more complicated and can stabilise an unstable system based on the equilibrium stability criterion in Equation 6, indicating more general criteria for stability must account for hydrodynamic stabilisation.

Figure $3 b$ shows the effect of decreasing the interfacial tension. For $\gamma=60 \mathrm{mN} / \mathrm{m}$, the bubble interactions are stable over a much wider range of the parameter space. The coalescence on approach region has disappeared, whilst the coalescence on retract region is much smaller. For this system, the critical interfacial tension defined 
using the equilibrium stability criterion (Equation 6) is $\gamma=60 \mathrm{mN} / \mathrm{m}$. Thus, at and below this limit all equilibrium interactions are stable and coalescence on approach is not possible. However, there is still a small coalescence on retract region, demonstrating that hydrodynamics can induce coalescence for stable equilibrium surface forces.

To demonstrate the sensitivity of the bubble collision outcome to the exact definition of the disjoining pressure, we present in Figure $3 c \& d$ the stability maps for the same bubble pair, this time with a retarded $\mathrm{vdW}$ force calculated using Lifshitz theory. The comparison between the resulting disjoining pressures is shown in Figure S1 in the SI. The non-retarded and retarded vdW give rise to similar DLVO disjoining pressures, with a small variation in the magnitude of the maximum repulsive pressure. However, the equilibrium stability criterion predicts that the retarded DLVO system will be stable for $\gamma<65 \mathrm{mN} / \mathrm{m}$ rather than $\gamma<60 \mathrm{mN} / \mathrm{m}$ for the non-retarded case. The stability maps in Figure 3 confirm this, and show that the coalescence regions are greatly reduced for the retarded case in comparison to the non-retarded case.

In Figure 4 we plot a three-dimensional stability map of the same bubble pair in Figure 3 a \& b, as a function of interaction velocity $U$ and interfacial tension $\gamma$ at three different values of overlap $X-h_{0}$. Here, all stable interactions are represented with white symbols, whilst the unstable interactions are shown with red and blue symbols representing coalescence on approach and retract, respectively. The vertical grey plane shows the critical interfacial tension based on the equilibrium stability criterion given in Equation 6: for $\gamma>60 \mathrm{mN} / \mathrm{m}$ the equilibrium interactions are unstable, and for $\gamma<60 \mathrm{mN} / \mathrm{m}$ equilibrium interactions are stable. For the lowest value of overlap shown, $X-h_{0}=2 \mu \mathrm{m}$, it is clear that most collisions are stable despite the pair being unstable at equilibrium. Thus, the interaction area between colliding bubbles needs to be significant for the equilibrium stability criterion to apply, and general stability criteria would need to reflect this. At the highest value of overlap shown, $X-h_{0}=10 \mu \mathrm{m}$, coalescence on retract can occur for interfacial tensions below the equilibrium stability limit, induced by hydrodynamic effects. As a consequence, generalised stability criteria would also need to account for this destabilising hydrodynamic mechanism.

To examine the transition from coalescence on approach to coalescence on retract, in Figure 5 we plot a contour map of the force at coalescence for the same bubble pair shown in Figure 3. The black line depicts the transition from coalescence on approach (to the left) to coalescence on retract (to the right). Also shown in Figure $5 \mathrm{~b}$ is a plot of the force at coalescence for fixed overlap of $X-h_{0}=10 \mu \mathrm{m}$ (the white dashed line on the contour map in Figure 5a)), along with the bubble shapes just before coalescence occurs. When the bubbles coalesce on approach, the force at coalescence is independent of overlap, because the film drains before the bubbles have been driven together completely. The region of high force near the transition line indicates that coalescence occurs at, or very close to, the end of the approach stage, where the maximum interaction force occurs. The high-force region immediately to the right of the black line signifies that coalescence occurs soon after the turn-around point, at the start of the retract stage. In this high force region the bubble forms a "dimple" at coalescence [61]. As the velocity increases further, the force at coalescence drops markedly (Figure 5). This indicates that the coalescence occurs much later in the retraction stage, far from the maximum interaction force experienced by the bubbles. 
In this region the bubble forms a "wimple"[45] at coalescence, which changes to a "nose" as the interaction velocity increases further. This nose formation is identical to the drop profiles formed by drops undergoing coalescence during separation in a microfluidic channel[49]. The marked drop in force at coalescence as the interaction velocity increases, and the corresponding change in bubble shape, suggests that there are two distinct mechanisms leading to coalescence on retract.

In order to further elucidate the mechanisms of coalescence, we plot the time history of the interaction force, the minimum separation within the film, and the corresponding hydrodynamic pressure at the point of minimum separation in Figure 6. Three mechanisms of coalescence are evident. The first mechanism leads to coalescence on approach, and is clear when examining the $U=10 \mu \mathrm{m} / \mathrm{s}$ and $25 \mu \mathrm{m} / \mathrm{s}$ cases. The bubbles are moving slowly enough such that the film completely drains on approach, and coalescence occurs before the bubbles reach the point of maximum interaction. The second mechanism, shown in the $U=50 \mu \mathrm{m} / \mathrm{s}$ example, results in coalescence on retract. In this case, the film does not have time to drain completely on the approach stage, but continues to drain during the initial part of the approach stage. At this intermediate velocity the drops are not moving fast enough apart to counteract the film drainage and coalescence occurs. For this case the minimum separation decreases monotonically before coalescence occurs in the initial part of the retract stage. As the interaction velocity is increased further to $U=60 \mu \mathrm{m} / \mathrm{s}$ a third mechanism of coalescence becomes evident. The film drains continuously during the approach stage, and the beginning of the retract stage, but then briefly begins to thicken as the bubbles move apart fast enough to avoid complete drainage occurring. However, the high velocity induces a negative hydrodynamic pressure within the film at the point of minimum separation, which reverses the film thickening and drives the bubbles to coalescence. At very high drive velocities $(U=200 \mu \mathrm{m} / \mathrm{s})$, the film again thins monotonically to coalescence because the negative hydrodynamic pressure occurs earlier in the retract stage, before the film has time to thicken. As the velocity increases further $(U=250 \mu \mathrm{m} / \mathrm{s})$, the drive velocity becomes large enough to move the bubbles apart completely before the negative hydrodynamic pressure can thin the film sufficiently, and coalescence does not occur.

In Figure 6a we plot a stability map of the same bubble pair at $\gamma=70 \mathrm{mN} / \mathrm{m}$, but for a higher salt concentration of $10 \mathrm{mM}$. At this concentration the Debye length is $\sim 3$ $\mathrm{nm}$, and the EDL repulsion is much less than that for the $0.1 \mathrm{mM}$ case considered above. For this particular system, the critical interfacial tension based on the equilibrium stability criterion of Equation 6 is extremely low, and thus this system is almost always unstable at equilibrium. As a consequence, coalescence occurs over the entirety of the parameter space studied, with a significantly larger coalescence on approach region that the $0.1 \mathrm{mM}$ system. The position of the line delineating coalescence on approach to coalescence on retract as the interfacial tension is decreased from $\gamma=70$ to $20 \mathrm{mN} / \mathrm{m}$ is shown in Figure 6b). As $\gamma$ decreases, the interactions remain unstable, but the transition from coalescence on approach to coalescence on retract occurs at smaller approach velocities.

Other surface forces will result in stable or unstable interactions with similar interplay with hydrodynamic drainage effects. The subtleties of each surface forces are interesting, but the overall behaviours are commensurate with the discussion for Figures 3-7. In the SI we demonstrate that a bubble pair with a depletion surface 
force also gives significant coalescence on approach and coalescence on retract regions due to its inherently attractive nature. We also show that a drop pair with a (repulsive) steric force does not result in coalescence over the parameter space examined.

In Figure 7 we consider interactions of the same bubble pair system as in Figure $3 \mathrm{a}$ and $b$, this time with an approach and hold (or dwell) drive profile, mimicking drop interactions produced using cantilevered or translated capillaries, or through buoyant collisions. At this interfacial tension the interactions are unstable at equilibrium, and thus will coalesce after being held together at some finite time. Based on the literature approach to drop coalescence, we define coalescence time to be the time taken for the drops to coalesce in the dwell stage. There are many theoretical approaches showing that the coalescence time for a drop pair or a drop and a flat interface interacting at a constant applied force $\mathrm{F}$ varies as

$$
t_{\text {coalescence }} \sim \frac{F^{a} R^{b} \mu}{\gamma^{c} A_{H}{ }^{d}}
$$

where $A_{H}$ is the Hamaker constant, and $a, b, c \& d$ are scaling exponents. The exponents range from $1 / 4<a<0.84,31 / 24<b<7 / 4,1.14<c<1.38$ and $2 / 5<d<$ $4 / 7$ [72-78]. Inherent in the derivation of these scaling relations is that the approach velocity of the drops plays no role in the time to coalescence. However, our results for a bubble pair interacting in water (i.e. for fixed Hamaker constant and interfacial tension) show that the approach velocity affects the time to coalescence considerably, and that a simple scaling relation is not present. For this particular system, the ratio of the coalescence time to the bubble approach time is $O(1)$, demonstrating that initial conditions are important. We also show results for systems with viscosity one and two orders of magnitude higher, approximating the viscosities of the systems observed with cantilevered capillaries[79]. Here the coalescence times scale linearly with viscosity for moderate to high forces, and are consequently one and two orders of magnitude higher respectively. For these systems, the ratio of approach time to coalescence time is $O(10)$ and $O(100)$, respectively. Therefore, there is no dependence upon the initial conditions, and a scaling similar to Equation 8 is present for moderate to high forces. Interestingly, however, the behaviour of the highviscosity system for low forces displays non-monotonic behaviour not accessible using scaling analysis.

As a consequence, it is clear that for micron-sized bubbles in water, and micro-drops in moderate viscosity oils, that the ratio of approach time to coalescence time will in general be $O(1)$, and scaling analyses will not give accurate descriptions of the coalescence behaviour due to the dependence upon initial conditions. For larger sized drops and bubbles, higher viscosity continuous media, or systems with large density differences, scaling analyses do provide reasonable predictions of coalescence time for moderate to high forces. This context is critical when making general statements about drop and bubble coalescence, keeping in mind that, in practice, the continuous phase viscosity in an emulsion or foam may span this entire viscosity range depending on the point in processing or formulation. 


\section{Conclusions}

We have used an extensively validated numerical model that accounts for drop deformation, equilibrium surface forces and hydrodynamic drainage to determine the precise conditions under which micron-sized drops or bubbles will undergo coalescence. We have considered two types of collision events: a linear approach and retract profile mimicking AFM experiments, and a linear approach and hold profile similar to constant force (e.g. buoyant) collisions. The parameter space examined includes interaction velocity, the amount of drop "overlap" (a measure of the extent of the collision), interfacial tension, and salt concentration. We have shown that the a well-defined description of the equilibrium surface force as a function of film thickness (e.g. DLVO forces) can be used to make precise predictions of drop or bubble collision stability when hydrodynamic effects are negligible. Specifically, if the Laplace pressure $2 \gamma / R$ of a drop or a bubble is greater than the disjoining pressure for all possible film thicknesses, then the interaction is unstable and coalescence will result. This equilibrium stability criterion assumes that the collision is significant enough that the film drains enough for attractive surface forces (e.g. van der Waals) to induce coalescence.

Using the aforementioned numerical model, we have defined the specific conditions when hydrodynamic effects can render unstable equilibrium collisions stable, primarily due to high interaction velocities. Further, we have mapped the precise conditions whereby stable equilibrium collisions can become unstable due to negative hydrodynamic pressure within the film, resulting coalescence whilst the drop or bubble pairs are undergoing separation, observed experimentally in microfluidics[49], in a four-roll mill [48], and in AFM experiments [28]. In summary, general stability criteria describing drop or bubble collisions must account for hydrodynamic stabilisation due to high interaction velocities, and destabilisation on separation induced by negative hydrodynamic pressure in the film. Lastly, we have established that initial conditions are important, and consequently scaling analyses are invalid, for constant force collisions when the coalescence time is of the same order as the approach time, true for micron-sized bubbles or drops suspended in moderate viscosity media.

One limitation to the approach used in this study requires a well-defined model of the surface force as a function of the film thickness. In the case of the hydrophobic force, there is now some clarity in the literature, where for drops or bubbles we have validated experimental force laws[42, 80], with more work and validation ongoing for rigid surfaces[81]. Yet, the recent work by Abbott and co-workers[82] in probing a surface coated with synthetic peptides suggests that the hydrophobic force for may be more complicated and a subsequent description of the force as a function of separation may remain elusive. In addition, the other area where this model is limited in in the area of specific ion effects. There are AFM data for simple systems, in the absence of any surfactant for both polar [83] and non-polar oils[84], suggesting there are surface force effects that have yet to be captured in a reproducible way, hindering efforts to describe a well-defined surface force model.

The dynamic nature of the interactions for this work have been limited to assume that the interfacial deformation can be described using the Laplace equation, neglecting any interfacial rheological behaviour. For most of the AFM systems studied 
previously, this has been the case, but an interesting study by Gunning et al. has showcased how there can be more complex behaviour when the interface has a relaxation time associated with its response to deformation [41] where more study is warranted in this area. 


\section{References}

[1] S.L. Carnie, D.Y.C. Chan, C. Lewis, R. Manica, R.R. Dagastine, Langmuir 21 (2005) 2912.

[2] N. Bremond, A.R. Thiam, J. Bibette, Physical Review Letters 100 (2008) 1.

[3] H. Chen, J. Li, Tribology Online 11 (2016) 281.

[4] A.R. Abate, T. Hung, R.A. Sperling, P. Mary, A. Rotem, J.J. Agresti, M.A.

Weiner, D.A. Weitz, Lab on a chip 13 (2013) 4864.

[5] T.P. Lagus, J.F. Edd, Journal of Physics D: Applied Physics 46 (2013) 114005.

[6] S.E. Kentish, G.W. Stevens, Chem. Eng. J. 84 (2001) 149.

[7] V. Bergeron, Current Opinion in Colloid \& Interface Science 4 (1999) 249.

[8] V. Bergeron, C.J. Radke, Langmuir 8 (1992) 3020.

[9] D. Exerowa, D. Kashchiev, D. Platikanov, Advances in Colloid and Interface Science 40 (1992) 201.

[10] A. Sheludko, Adv. Colloid Interface Sci. 1 (1967) 391.

[11] K.J. Mysels, M.N. Jones, Discussions of the Faraday Society 42 (1966) 42.

[12] A.D. Nikolov, D.T. Wasan, Journal of Colloid and Interface Science 133

(1989) 1.

[13] K. Kumar, A.D. Nikolov, D.T. Wasan, Journal of Colloid and Interface Science 256 (2002) 194.

[14] R. Aveyard, B.P. Binks, W.G. Cho, L.R. Fisher, P.D.I. Fletcher, F. Klinkhammer, Langmuir 12 (1996) 6561.

[15] J.N. Connor, R.G. Horn, Faraday Discussions 123 (2003) 193.

[16] R.G. Horn, D.J. Bachmann, J.N. Connor, S.J. Miklavcic, Journal of Physics:

Condensed Matter 8 (1996) 9483.

[17] R.A. Pushkarova, R.G. Horn, Colloids and Surfaces A: Physicochemical and Engineering Aspects 261 (2005) 147.

[18] H.-J. Butt, Journal of Colloid and Interface Science 166 (1994) 109.

[19] W.A. Ducker, Z. Xu, J.N. Israelachvili, Langmuir 10 (1994) 3279.

[20] M.L. Fielden, R.A. Hayes, J. Ralston, Langmuir 12 (1996) 3721.

[21] D.E. Aston, J.C. Berg, Journal of Pulp and Paper Science 24 (1998) 121.

[22] P.G. Hartley, F. Grieser, P. Mulvaney, G.W. Stevens, Langmuir 15 (1999)

7282.

[23] P. Mulvaney, J.M. Perera, S. Biggs, F. Grieser, G.W. Stevens, Journal of Colloid and Interface Science 183 (1996) 614.

[24] B.A. Snyder, D.E. Aston, J.C. Berg, Langmuir 13 (1997) 590.

[25] R.R. Dagastine, R. Manica, S.L. Carnie, D.Y.C. Chan, G.W. Stevens, F. Grieser, Science (Washington, DC, United States) 313 (2006) 210.

[26] R.R. Dagastine, G.W. Stevens, D.Y.C. Chan, F. Grieser, Journal of Colloid and Interface Science 273 (2004) 339.

[27] A.P. Gunning, A.R. Mackie, P.J. Wilde, V.J. Morris, Langmuir 20 (2004) 116. [28] I.U. Vakarelski, R. Manica, X.S. Tang, S.J. O'Shea, G.W. Stevens, F. Grieser, R.R. Dagastine, D.Y.C. Chan, Proc. Natl. Acad. Sci. U. S. A. 107 (2010) 11177.

[29] C. Shi, X. Cui, L. Xie, Q.X. Liu, D.Y.C. Chan, J.N. Israelachvili, H.B. Zeng, ACS Nano 9 (2015) 95.

[30] L.X. Wang, D. Sharp, J. Masliyah, Z.H. Xu, Langmuir 29 (2013) 3594.

[31] J.M. Frostad, M.C. Collins, L.G. Leal, Langmuir 29 (2013) 4715.

[32] D.E. Aston, J.C. Berg, Journal of Colloid and Interface Science 235 (2001) 
[33] H.J. Lockie, R. Manica, G.W. Stevens, F. Grieser, D.Y.C. Chan, R.R. Dagastine, Langmuir 27 (2011) 2676.

[34] R.F. Tabor, R. Manica, D.Y.C. Chan, F. Grieser, R.R. Dagastine, Physical Review Letters 106 (2011) 064501.

[35] R.F. Tabor, C. Wu, H. Lockie, R. Manica, D.Y.C. Chan, F. Grieser, R.R. Dagastine, Soft Matter 7 (2011) 8977.

[36] A. Gromer, R. Penfold, A.P. Gunning, A.R. Kirby, V.J. Morris, Soft Matter 6 (2010) 3957.

[37] O. Manor, T.T. Chau, G.W. Stevens, D.Y.C. Chan, F. Grieser, R.R. Dagastine, Langmuir 28 (2012) 4599.

[38] C.J. Fewkes, R.F. Tabor, R.R. Dagastine, Soft Matter 11 (2015) 1303.

[39] R.F. Tabor, D.Y.C. Chan, F. Grieser, R.R. Dagastine, Journal of Physical Chemistry Letters 2 (2011) 434.

[40] C. Browne, R.F. Tabor, F. Grieser, R.R. Dagastine, Journal of Colloid and Interface Science 449 (2014) 236.

[41] A.P. Gunning, A.R. Kirby, P.J. Wilde, R. Penfold, N.C. Woodward, V.J. Morris, Soft Matter 9 (2013) 11473.

[42] R.F. Tabor, C. Wu, F. Grieser, R.R. Dagastine, D.Y.C. Chan, Journal of Physical Chemistry Letters 4 (2013) 3872.

[43] R.F. Tabor, F. Grieser, R.R. Dagastine, D.Y.C. Chan, Journal of Colloid and Interface Science 371 (2012) 1.

[44] D.E. Aston, J.C. Berg, Industrial \& Engineering Chemistry Research 41 (2002) 389.

[45] L.Y. Clasohm, J.N. Connor, O.I. Vinogradova, R.G. Horn, Langmuir 21 (2005) 8243.

[46] E. Klaseboer, J.P. Chevaillier, C. Gourdon, O. Masbernat, Journal of Colloid and Interface Science 229 (2000) 274.

[47] L.Y. Clasohm, I.U. Vakarelski, R.R. Dagastine, D.Y.C. Chan, G.W. Stevens, F. Grieser, Langmuir 23 (2007) 9335.

[48] M. Borrell, Y. Yoon, L.G. Leal, Physics of Fluids 16 (2004) 3945.

[49] N. Bremond, A.R. Thiam, J. Bibette, Physical Review Letters 100 (2008) $024501 / 1$.

[50] D.Z. Gunes, X. Clain, O. Breton, G. Mayor, A.S. Burbidge, Journal of Colloid and Interface Science 343 (2010) 79.

[51] R.F. Tabor, C. Wu, F. Grieser, D.Y.C. Chan, R.R. Dagastine, Soft Matter 9 (2013) 2426.

[52] J.M. Frostad, A. Paul, L.G. Leal, Bulletin of the American Physical Society 59 (2014) BAPS.2014.DFD.L13.8.

[53] S.A. Edwards, S.L. Carnie, O. Manor, D.Y.C. Chan, Langmuir 25 (2009) 3352.

[54] R.R. Dagastine, G.B. Webber, R. Manica, G.W. Stevens, F. Grieser, D.Y.C. Chan, Langmuir 26 (2010) 11921.

[55] R. Pillai, J. Berry, D. Harvie, M. Davidson, Chemical Engineering Science (2016).

[56] R. Pillai, J.D. Berry, D.J. Harvie, M.R. Davidson, in: (Ed.)^(Eds.)11th International Conference on CFD in the Minerals and Process Industries, Melbourne, 2015.

[57] J.D. Berry, M.R. Davidson, D.J.E. Harvie, Journal of Computational Physics 251 (2013) 209. 
[58] R. Pillai, J.D. Berry, D.J.E. Harvie, M.R. Davidson, International Journal of Computational Methods and Experimental Measurements 4 (2016) 33.

[59] D. Bhatt, J. Newman, C.J. Radke, Langmuir 17 (2001) 116.

[60] D.Y.C. Chan, R.R. Dagastine, L.R. White, Journal of Colloid and Interface Science 236 (2001) 141.

[61] D.Y.C. Chan, E. Klaseboer, R. Manica, Advances in Colloid and Interface Science 165 (2011) 70.

[62] M. Shahalami, L.X. Wang, C. Wu, J.H. Masliyah, Z.H. Xu, D.Y.C. Chan, Advances in Colloid and Interface Science 217 (2015) 31.

[63] R. Manica, J.N. Connor, S.L. Carnie, R.G. Horn, D.Y.C. Chan, Langmuir FIELD Full Journal Title:Langmuir 23 (2007) 626.

[64] R. Manica, J.N. Connor, R.R. Dagastine, S.L. Carnie, R.G. Horn, D.Y.C. Chan, Physics of Fluids 20 (2008) 032101/1.

[65] R.F. Tabor, A.J. Morfa, F. Grieser, D.Y.C. Chan, R.R. Dagastine, Langmuir 27 (2011) 6026.

[66] G. Gillies, C.A. Prestidge, P. Attard, Langmuir 18 (2002) 1674.

[67] S.L. Carnie, D.Y.C. Chan, R. Manica, ANZIAM J 46(E) (2005) C805.

[68] R.F. Tabor, D.Y.C. Chan, F. Grieser, R.R. Dagastine, Angewandte Chemie 123 (2011) 3516.

[69] R.R. Dagastine, D.C. Prieve, L.R. White, Journal of Colloid and Interface Science 231 (2000) 351.

[70] V.A. Parsegian, G.H. Weiss, Journal of Colloid and Interface Science 81 (1981) 285.

[71] I.B. Ivanov, K.D. Danov, P.a. Kralchevsky, Colloids and Surfaces A:

Physicochemical and Engineering Aspects 152 (1999) 161.

[72] J.-D. Chen, P.S. Hahn, J.C. Slattery, AIChE Journal 30 (1984) 622.

[73] K.B. Fu, J.C. Slattery, Journal of Colloid and Interface Science 315 (2007) 569.

[74] E.S. Basheva, T.D. Gurkov, I.B. Ivanov, G.B. Bantchev, B. Campbell, R.P. Borwankar, Langmuir 15 (1999) 6764.

[75] J.M. Frostad, J. Walter, L.G. Leal, Physics of Fluids 25 (2013).

[76] M.B. Nemer, X. Chen, D.H. Papadopoulos, J. Bławzdziewicz, M.

Loewenberg, Journal of Colloid and Interface Science 308 (2007) 1.

[77] A.K. Chesters, Chemical Engineering Research and Design 69 (1991) 259.

[78] G.E. Charles, S.G. Mason, Journal of Colloid Science 15 (1960) 236.

[79] J.M. Frostad, A. Paul, L.G. Leal, Physical Review Fluids 1 (2016) 033904.

[80] R.F. Tabor, F. Grieser, R.R. Dagastine, D.Y.C. Chan, Phys. Chem. Chem. Phys. $16(2014) 18065$.

[81] S.H. Donaldson, A. Royne, K. Kristiansen, M.V. Rapp, S. Das, M.A. Gebbie, D.W. Lee, P. Stock, M. Valtiner, J. Israelachvili, Langmuir 31 (2015) 2051.

[82] C.D. Ma, C.X. Wang, C. Acevedo-Velez, S.H. Gellman, N.L. Abbott, Nature 517 (2015) 347.

[83] R.R. Dagastine, T.T. Chau, D.Y.C. Chan, G.W. Stevens, F. Grieser, Faraday Discussions 129 (2005) 111.

[84] H. Lockie, R. Manica, R.F. Tabor, G.W. Stevens, F. Grieser, D.Y.C. Chan, R.R. Dagastine, Langmuir 28 (2012) 4259. 

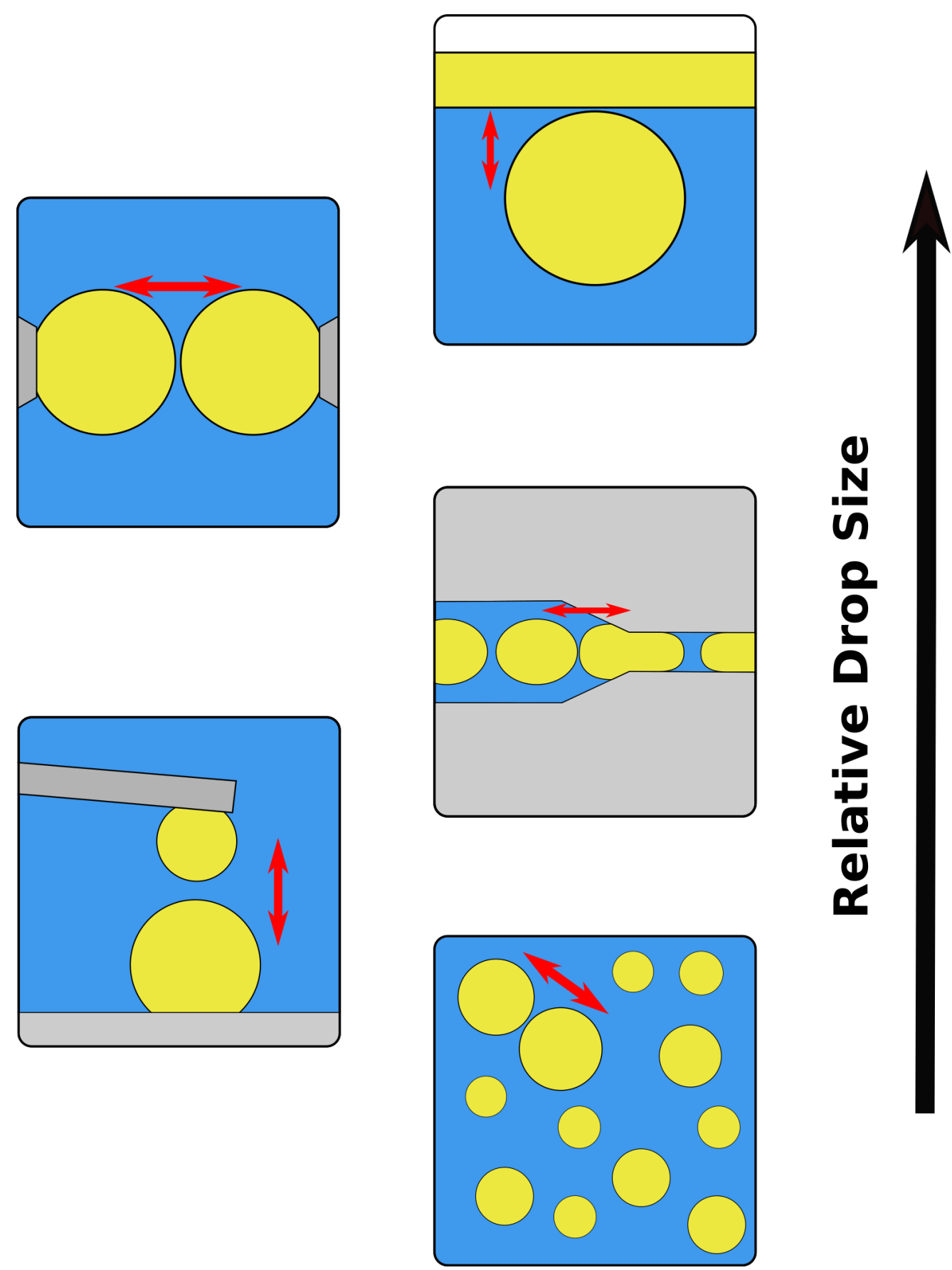

Figure 1 Schematic of systems in which drops or bubbles can undergo coalescence. The systems are arranged in terms of relative drop or bubble size from smallest to largest: emulsions (sub-micron radii) and foams (10s of microns), AFM measurements ( $\sim 5$ microns to several hundred microns), microfluidic devices (10s to 100 s of microns, drops fixed on capillaries ( $>100$ s of microns), and drop collision with an interface ( $\mathrm{mm}$ radii and larger). 


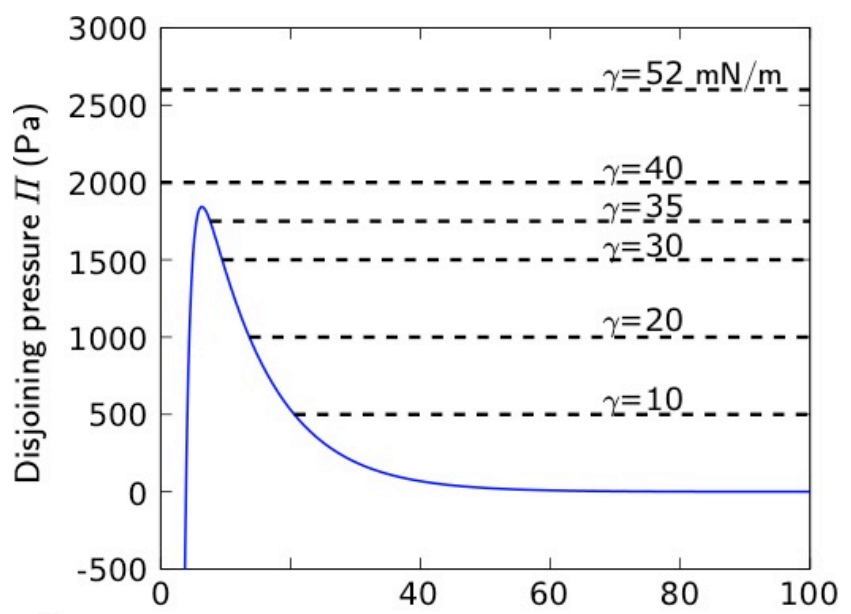

a)

Film thickness $h(\mathrm{~nm})$

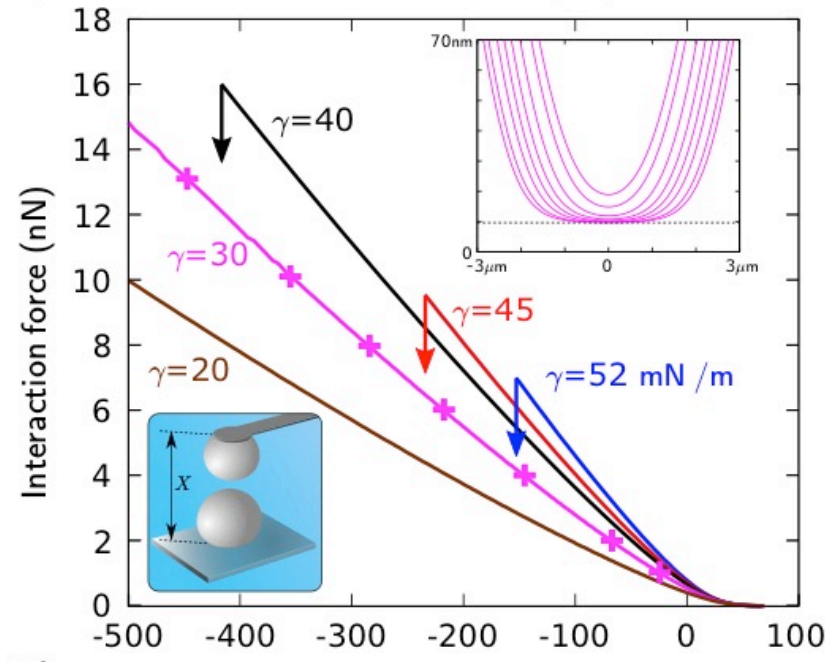

b) Distance $\Delta X(\mathrm{~nm})$

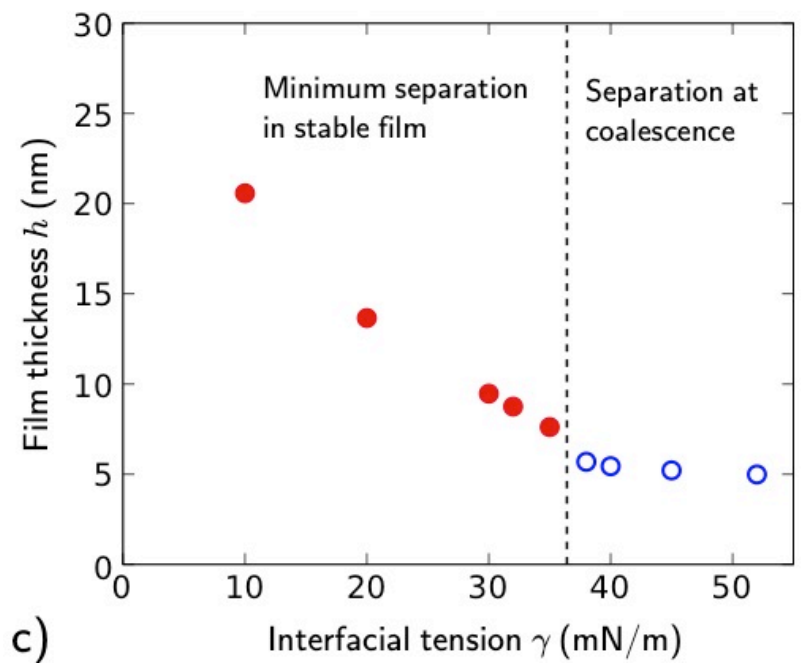

Figure 2 a) DLVO disjoining pressure between two flat tetradecane interfaces adjacent to a binary electrolyte, with a surface potential of $-17 \mathrm{mV}$ and an ionic strength of $1 \mathrm{mM}$. The dashed lines indicate the Laplace pressures of a drop of radius $R=40 \mu \mathrm{m}$ at different interfacial tensions. b) Equilibrium interactions of two tetradecane drops of radii $R=40 \mu \mathrm{m}$ driven together over a distance $\Delta X$ at different interfacial tensions in the presence of the DLVO force described in a), calculated using the Chan-Dagastine-White model. The arrows denote the point at which coalescence occurs. The inset shows the stable film profiles corresponding to the + symbols shown on the interfacial tension $\boldsymbol{\gamma}=\mathbf{3 0} \mathrm{mN} / \mathrm{m}$ curve. c) The minimum stable film thickness (red solid symbols) and the minimum film thickness prior to coalescence (blue open symbols) for the same system as b). The dashed line delineates the critical interfacial tension where the system becomes unstable. 

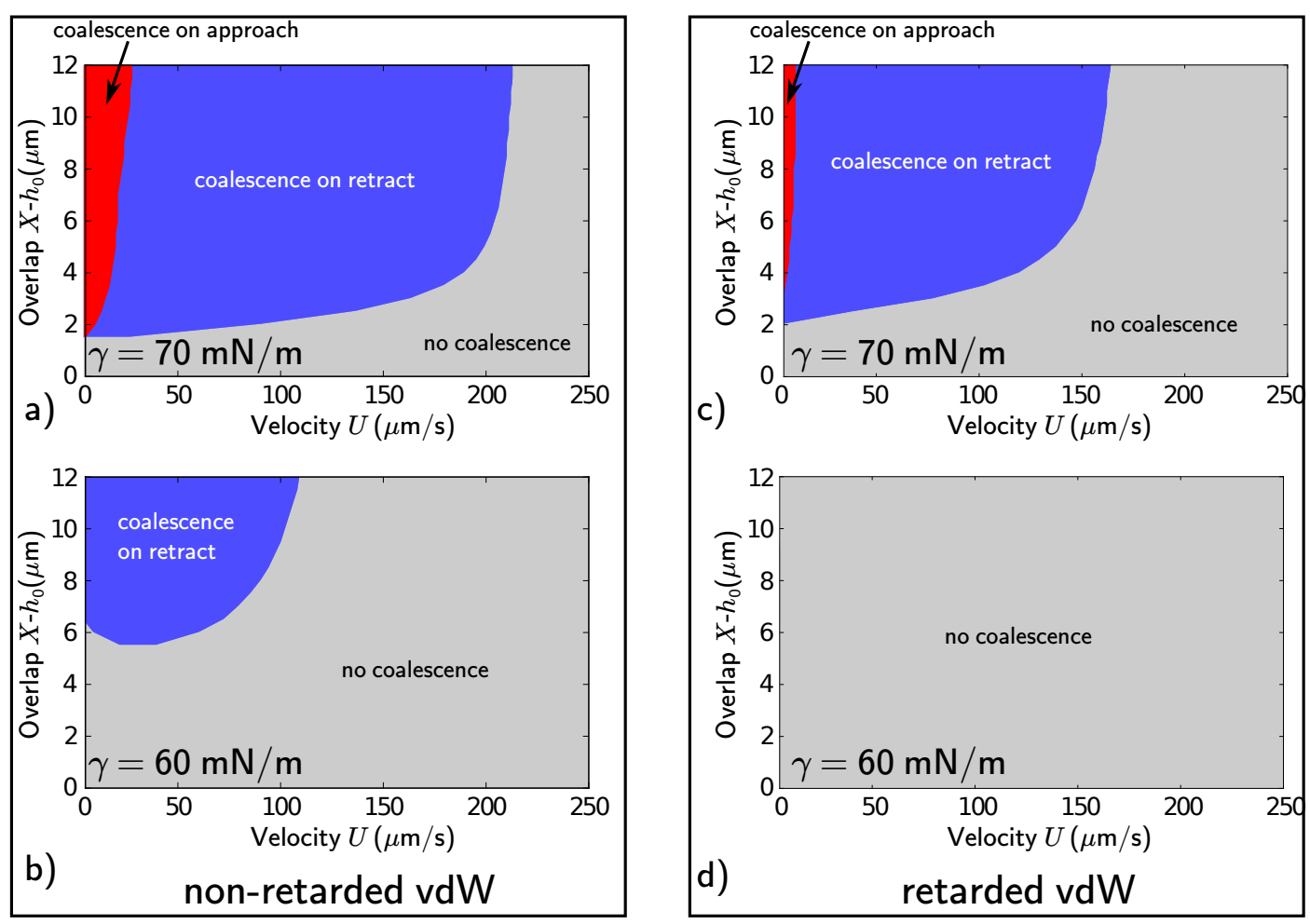

Figure 3 Stability maps of the interaction between two air bubbles in water containing $0.1 \mathrm{mM}$ salt solution for varying drive velocities $\boldsymbol{U}$ and overlaps $\boldsymbol{X}-\boldsymbol{h}_{\mathbf{0}}$. The left pane shows the stability maps calculated using a non-retarded vdW for two interfacial tensions: a) $70 \mathrm{mN} / \mathrm{m}$ and b) $60 \mathrm{mN} / \mathrm{m}$. The right pane shows the stability maps calculated using a retarded vdW for the same two interfacial tensions: c) $70 \mathrm{mN} / \mathrm{m}$ and d) $60 \mathrm{mN} / \mathrm{m}$. The red and blue regions indicate coalescence on approach and retract respectively, while the grey region represents stable interactions (where no coalescence occurs). The bubbles are initially separated by a distance $\boldsymbol{h}_{\mathbf{0}}$, before being driven together over a distance $\boldsymbol{X}=20 \mu \mathrm{m}$ with velocity $\boldsymbol{U}$, and then pulled apart with velocity $-\boldsymbol{U}$. The bubble radii $\boldsymbol{R}_{\mathbf{1}}$ and $\boldsymbol{R}_{\mathbf{2}}$ are 59.5 and 45 um respectively, and the surface potential of the air-water interface is $-72 \mathrm{mV}$. All other parameters are listed in Table S3. The retarded vdW is calculated using Lifshitz theory. 


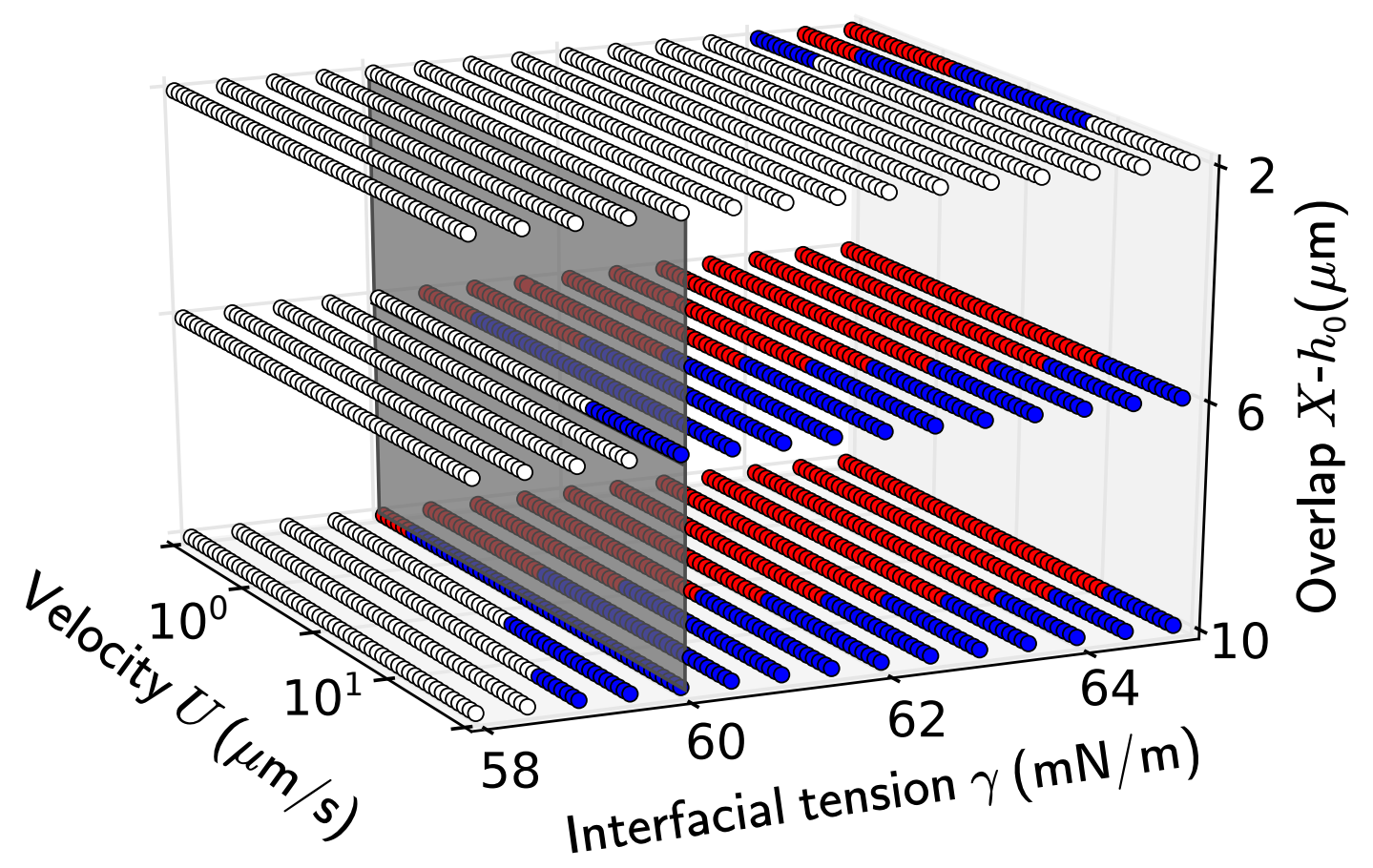

Figure 3 Stability map of the interaction between two air bubbles in water containing $0.1 \mathrm{mM}$ salt solution for varying drive velocities, bubble deformation, and interfacial tensions. The red and blue markers indicate coalescence on approach and retract respectively, while the white markers represent stable interactions (where no coalescence occurs). The vertical grey plane represents the critical equilibrium interfacial tension, below (above) which all equilibrium interactions are stable (unstable) for this bubble pair. The bubbles are initially separated by a distance $\boldsymbol{h}_{\mathbf{0}}$, before being driven together over a distance $\boldsymbol{X}=20 \mu \mathrm{m}$ with velocity $\boldsymbol{U}$, and then pulled apart with velocity $-\boldsymbol{U}$. The bubble radii $\boldsymbol{R}_{\mathbf{1}}$ and $\boldsymbol{R}_{\mathbf{2}}$ are 59.5 and $45 \mu \mathrm{m}$ respectively, and the surface potential of the air-water interface is $-72 \mathrm{mV}$. All other parameters are listed in Table S3. 

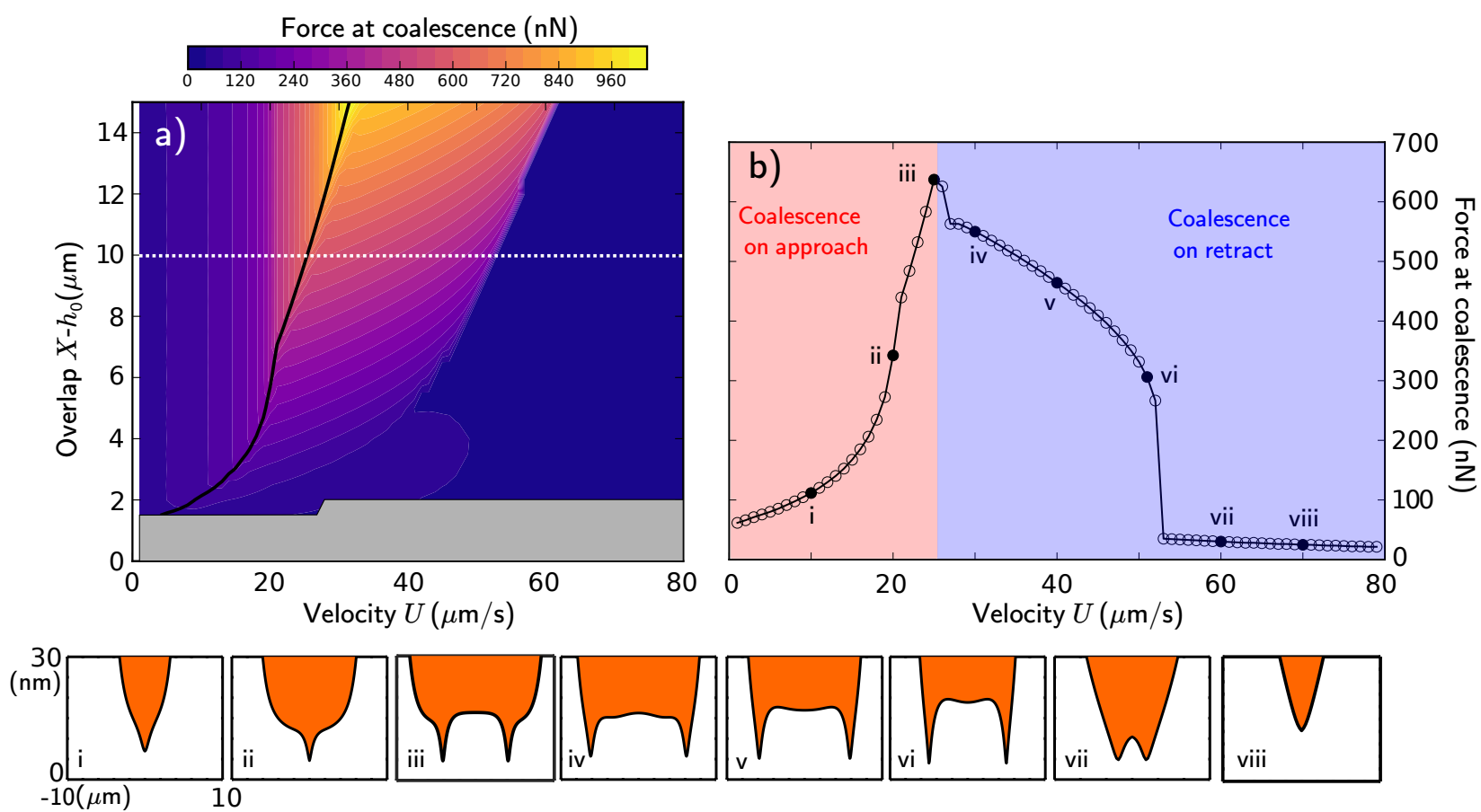

Figure 4 Force and film profiles at coalescence for the interaction between two air bubbles in water containing $0.1 \mathrm{mM}$ salt solution with interfacial tension $70 \mathrm{mN} / \mathrm{m}$. a) Contour map of the force at coalescence for varying approach velocities and overlaps. The black line delineates the boundary between coalescence on approach (left) and coalescence on retract (right). The white dashed line indicates the values plotted in $b$ ), and the grey region represents stable interactions. b) Force at coalescence over a range of approach velocities for fixed overlap $\boldsymbol{X}-\boldsymbol{h}_{\mathbf{0}}=\mathbf{1 0} \boldsymbol{\mu m}$. The corresponding film profiles at coalescence are shown in the panels. The bubbles are initially separated by a distance $\boldsymbol{h}_{\mathbf{0}}$, before being driven together over a distance $\boldsymbol{X}=20 \mu \mathrm{m}$ with velocity $\boldsymbol{U}$, and then pulled apart with velocity $-\boldsymbol{U}$. The bubble radii $\boldsymbol{R}_{\mathbf{1}}$ and $\boldsymbol{R}_{\mathbf{2}}$ are 59.5 and $45 \mathrm{um}$ respectively, and the surface potential of the air-water interface is $-72 \mathrm{mV}$. All other parameters are listed in Table S3. 


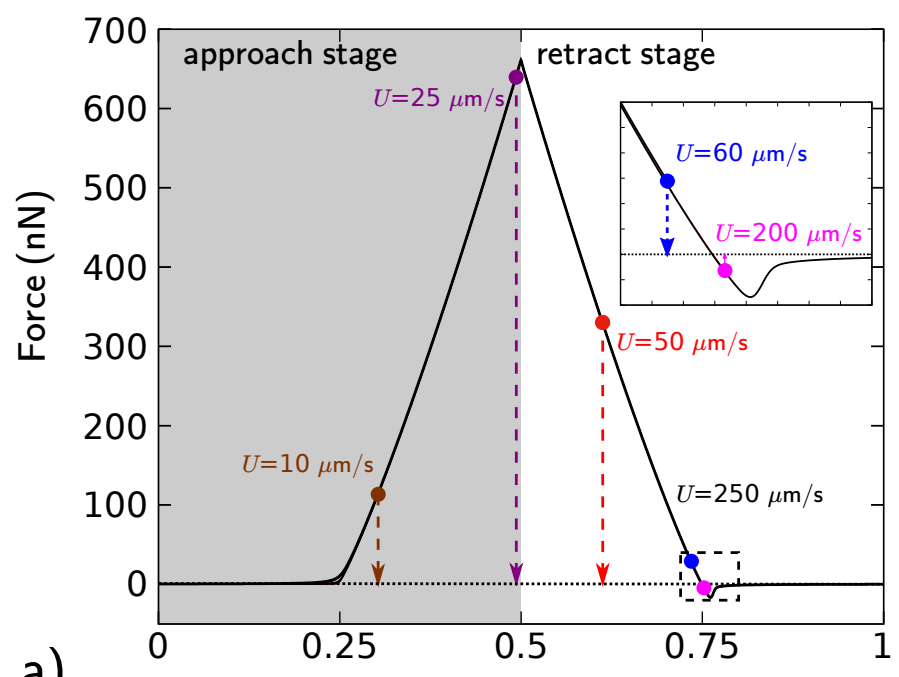

a)

$t V / X$

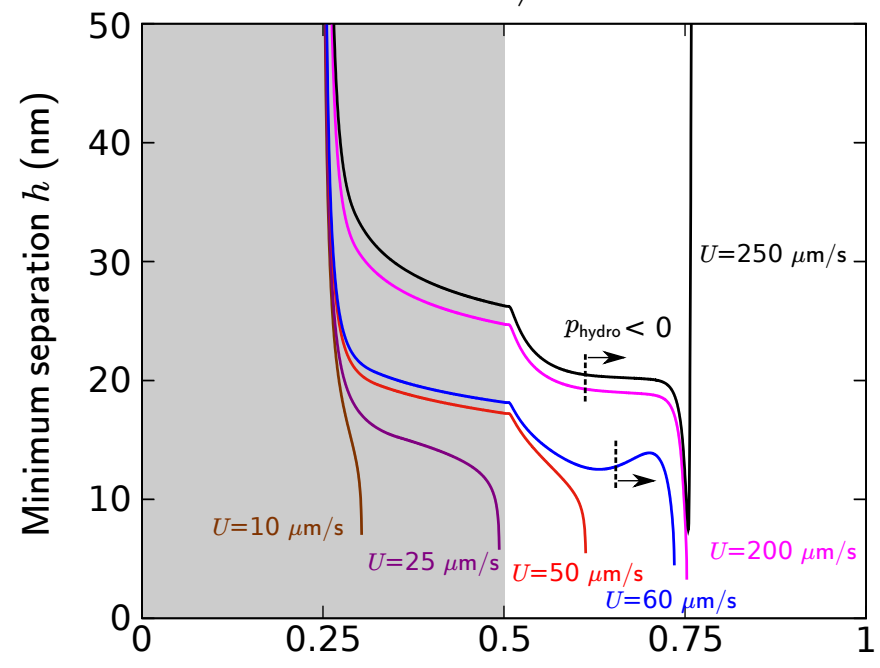

b)

$t V / X$

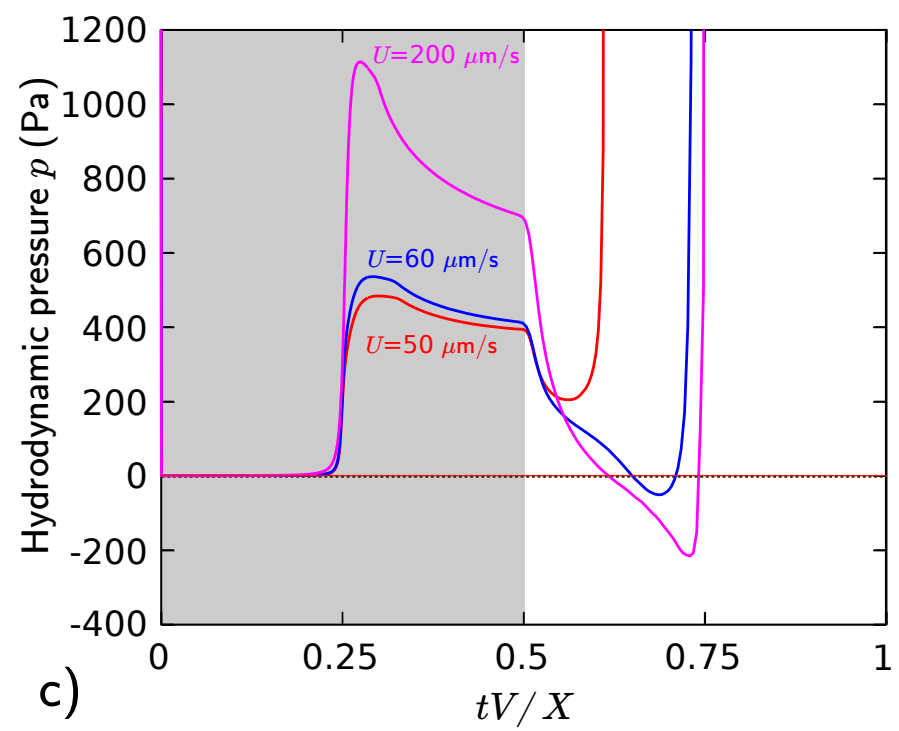

Figure 5 Time history of the interaction between two air bubbles in water containing 0.1 $\mathrm{mM}$ salt solution for fixed overlap $\boldsymbol{X}-\boldsymbol{h}_{\mathbf{0}}=\mathbf{1 0} \mu \mathrm{m}$. Time-dependent behaviour of the a) interaction force, $b$ ) hydrodynamic pressure at position of minimum separation, and c) the minimum separation, for varying drive velocities $U$ over the normalised time period $\mathrm{tV} / \mathrm{X}$. The filled circles depict the force at which the bubble collision for each velocity $U$ undergoes coalescence (except for the stable case at $U=250 \mu \mathrm{m} / \mathrm{s}$ ). The grey region represents the approach stage, and the white region represents the retract stage. The dashed lines in c) represent the time at which the hydrodynamic pressure at minimum separation becomes negative. 

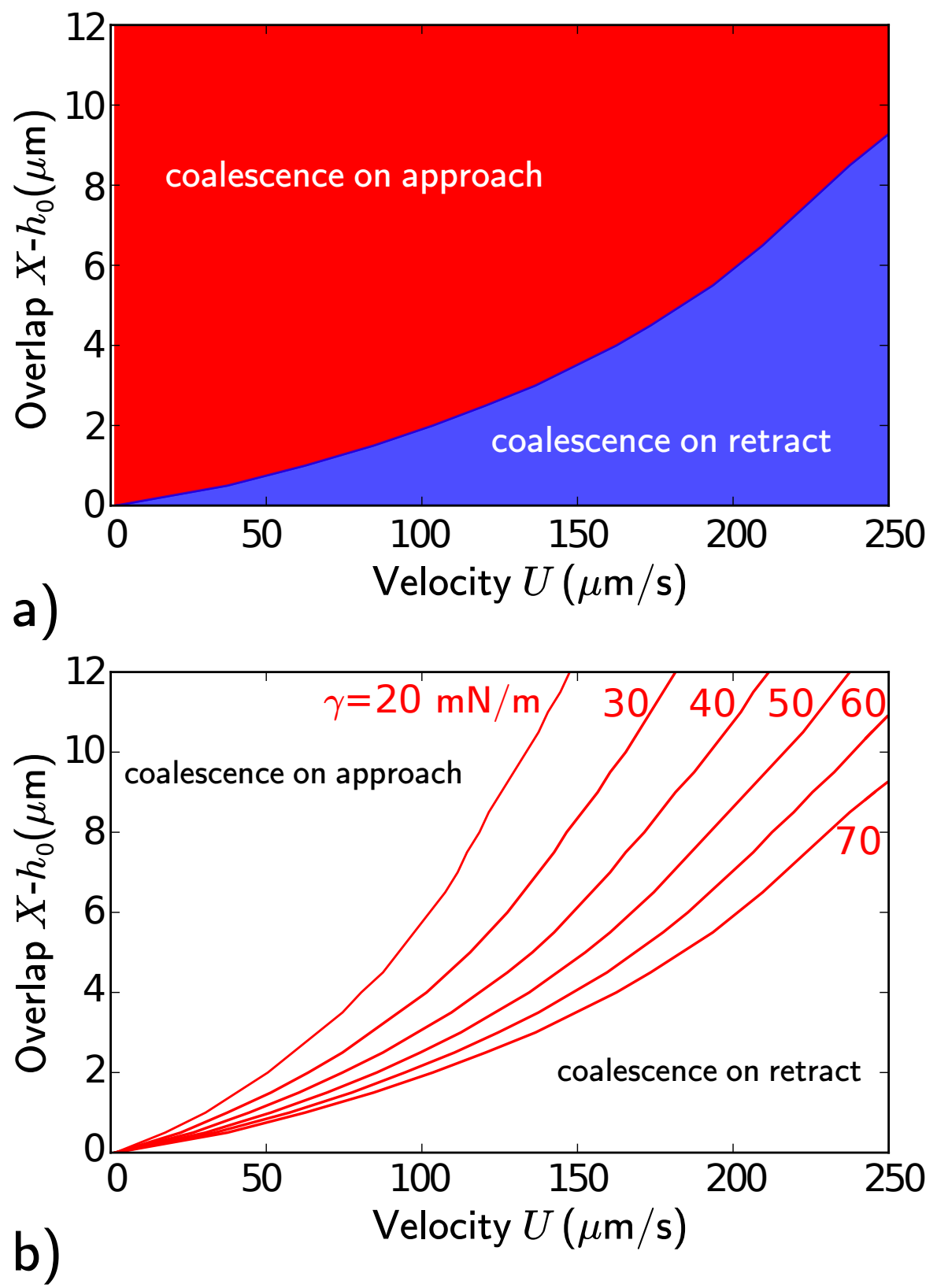

Figure 6 Interaction between two air bubbles in water containing $10 \mathrm{mM}$ salt solution for varying drive velocities $\boldsymbol{U}$ and overlap $\boldsymbol{X}-\boldsymbol{h}_{\mathbf{0}}$. a) Stability map for interfacial tension $70 \mathrm{mN} / \mathrm{m}$. The red and blue regions indicate coalescence on approach and retract respectively. b) Curves showing the boundary between coalescence on approach and coalescence on retract for varying interfacial tensions. The bubbles are initially separated by a distance $\boldsymbol{h}_{\mathbf{0}}$, before being driven together over a distance $\boldsymbol{X}=$ $20 \mu \mathrm{m}$ with velocity $\boldsymbol{U}$, and then pulled apart with velocity $-\boldsymbol{U}$. The bubble radii $\boldsymbol{R}_{\mathbf{1}}$ and $\boldsymbol{R}_{\mathbf{2}}$ are 59.5 and $45 \mu \mathrm{m}$ respectively, and the surface potential of the air-water interface is $-72 \mathrm{mV}$. All other parameters are listed in Table S3. 


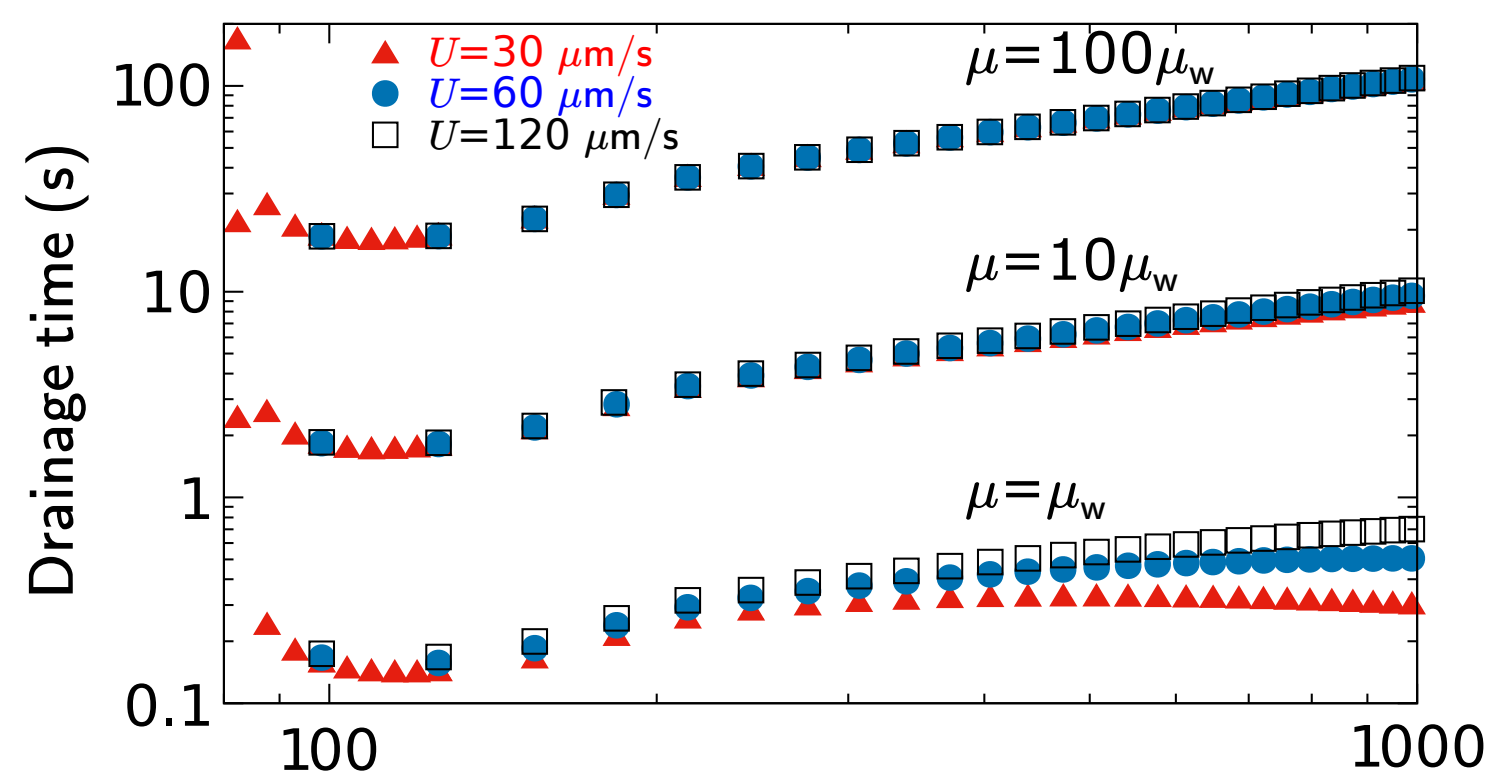

\section{Force at coalescence $(\mathrm{nN})$}

Figure 7 Drainage time versus force at coalescence for two air bubbles in a liquid containing $0.1 \mathrm{mM}$ salt solution, subjected to an approach and dwell interaction mimicking the constant force interactions of drops on cantilevered capillaries and buoyancy-driven collisions. The bubbles are initially separated by a distance $\boldsymbol{h}_{\mathbf{0}}$, before being driven together over a distance $\boldsymbol{X}=20 \mu \mathrm{m}$ with velocity $\boldsymbol{U}$, and then held until coalescence. The bubble radii $\boldsymbol{R}_{\mathbf{1}}$ and $\boldsymbol{R}_{\mathbf{2}}$ are 59.5 and 45 um respectively, the interfacial tension is $\gamma=65 \mathrm{mN} / \mathrm{m}$, and the surface potential of the air-water interface is $-72 \mathrm{mV}$. Three different values of viscosity are considered: $\boldsymbol{\mu}_{w}, 10 \boldsymbol{\mu}_{w}, \& 100 \mu_{w}$, where $\boldsymbol{\mu}_{\boldsymbol{w}}=\mathbf{8 . 9} \times \mathbf{1 0}^{-\mathbf{4}}$ Pa.s. All other parameters are listed in Table S3. 


\section{Mapping coalescence of micron-sized drops and bubbles}

Joseph D. Berry \& Raymond R. Dagastine

Department of Chemical and Biomolecular Engineering, University of Melbourne, Parkville VIC 3010, Australia.

Particulate Fluids Processing Centre, University of Melbourne, Parkville VIC 3010, Australia

SUPPLEMENTARY INFORMATION 
Table S1. Common surface forces and their corresponding interaction energies.

\begin{tabular}{|c|c|}
\hline Surface Force & Energy, $\boldsymbol{V}$ \\
\hline $\begin{array}{l}\text { Van der Waals } \\
\text { (noln-retarded) }\end{array}$ & $\begin{array}{l}\qquad V_{\mathrm{vdW}}=\frac{A_{H}}{12 \pi h^{2}} \\
A_{H} \text {, Hamaker constant }\end{array}$ \\
\hline Electric double layer & $\begin{array}{l}\quad V_{\mathrm{EDL}}=\frac{64 k_{B} T n^{0}}{\kappa} \tanh \left(\frac{e \psi_{o 1}}{4 k_{B} T}\right) \tanh \left(\frac{e \psi_{o 2}}{4 k_{B} T}\right) \exp (-\kappa h) \\
k_{B}, \text { Boltzmann constant; } T \text {, temperature; } n^{0}, \text { ion } \\
\text { concentration; } e \text {, elementary charge; } \psi_{o 1}, \text { surface potential; } \\
\kappa \text {, Debye length }\end{array}$ \\
\hline Structural & $\begin{array}{l}\qquad V_{\text {struct }}=A \exp \left(\frac{-h}{\epsilon}\right) \cos \left(\frac{2 \pi h}{\lambda}+\phi\right) \\
A \text {, amplitude fitting parameter } ; \epsilon \text {, decay length fitting } \\
\text { parameter; } \lambda \text {, periodicity fitting parameter; } \phi \text {, phase lag fitting } \\
\text { parameter }\end{array}$ \\
\hline Steric & $\begin{array}{l}\mathrm{V}_{\text {steric }}=\left\{\begin{array}{l}\alpha \frac{8 k_{B} T \delta}{35 s^{3}}\left[7\left(\frac{2 \delta}{h}\right)^{\frac{5}{4}}-5\left(\frac{h}{2 \delta}\right)^{\frac{7}{4}}-12\right] \\
\text { for } \mathrm{h}<2 \delta \\
\text { for } \mathrm{h} \geq 2 \delta\end{array}\right. \\
k_{B}, \text { Boltzmann constant; } T, \text { temperature; } \alpha, \quad \text { empirical } \\
\begin{array}{l}\text { coefficient; } \delta \text {, polymeric brush length; } s, \text { distance between } \\
\text { contact points on surface }\end{array}\end{array}$ \\
\hline Depletion & $\begin{array}{l}\quad \mathrm{V}_{\mathrm{dep}}= \begin{cases}\frac{\mathrm{B} \Pi_{\mathrm{osm}}}{2 R_{o}}(h-2 \Delta)\left(h+2 R_{o}\right) & \text { for } h<2 \Delta \\
0 & \text { for } h \geq 2 \Delta\end{cases} \\
\Pi_{\mathrm{osm}}=k_{B} T\left(\frac{C_{\text {poly }}}{A}+\frac{1}{\xi^{3}}\right) \\
k_{B}, \text { Boltzmann constant; } T, \text { temperature; } B, \text { partition } \\
\text { coefficient; } \Delta, \text { depletion layer thickness; } \Pi_{\text {osm }} \text { osmotic } \\
\text { pressure; } C_{\text {poly }} \text {, polyelectrolyte concentration; } A \text {, number of } \\
\text { monomers per charge; } \xi \text {, polyelectrolyte characteristic length }\end{array}$ \\
\hline
\end{tabular}


Table S2. Common surface forces and their corresponding disjoining pressures.

\begin{tabular}{|c|c|}
\hline Surface Force & Disjoining pressure, $\Pi=-\frac{\partial V}{\partial h}$ \\
\hline Van der Waals (non-retarded) & $\Pi_{\mathrm{vdW}}=-\frac{A_{H}}{6 \pi h^{3}}$ \\
\hline Electric double layer & $\Pi_{\mathrm{EDL}}=64 k_{B} T n^{0} \tanh \left(\frac{e \psi_{o 1}}{4 k_{B} T}\right) \tanh \left(\frac{e \psi_{o 2}}{4 k_{B} T}\right) \exp (-\kappa h)$ \\
\hline Structural & $\begin{array}{c}\Pi_{\text {struct }}=A \exp \left(\frac{-h}{\epsilon}\right)\left[\frac{1}{\epsilon} \cos \left(\frac{2 \pi h}{\lambda}+\phi\right)\right. \\
\left.+\frac{2 \pi}{\lambda} \sin \left(\frac{2 \pi h}{\lambda}+\phi\right)\right]\end{array}$ \\
\hline Steric & $\Pi_{\text {steric }}= \begin{cases}\alpha \frac{k_{B} T}{s^{3}}\left[\left(\frac{2 \delta}{h}\right)^{\frac{9}{4}}-\left(\frac{h}{2 \delta}\right)^{\frac{3}{4}}\right] & \text { for } \mathrm{h}<2 \delta \\
0 & \text { for } \mathrm{h} \geq 2 \delta\end{cases}$ \\
\hline Depletion & $\Pi_{\mathrm{dep}}=\left\{\begin{array}{lr}\frac{\mathrm{B} \Pi_{\mathrm{osm}}}{R_{o}}\left(\Delta-h-R_{o}\right) & \text { for } h<2 \Delta \\
0 & \text { for } h \geq 2 \Delta\end{array}\right.$ \\
\hline
\end{tabular}


Table S3. Properties of the bubble pair studied in Figures $3-8$.

\begin{tabular}{|l|l|}
\hline First drop radius $\mathrm{R}_{1}$ & $59.5 \mathrm{um}$ \\
\hline Second drop radius $\mathrm{R}_{2}$ & $45 \mathrm{um}$ \\
\hline Effective radius $\mathrm{R}$ & $51.2 \mathrm{um}$ \\
\hline First drop contact radius & $30 \mathrm{um}$ \\
\hline Second drop contact radius & $20 \mathrm{um}$ \\
\hline $\begin{array}{l}\text { Hamaker constant } \\
\text { (non-retarded vdW) }\end{array}$ & $-3.7211 \times 10^{-20} \mathrm{~J}$ \\
\hline Surface potential & $-72 \mathrm{mV}(\mathrm{C}=0.1 \mathrm{mM})$ \\
\hline Viscosity & $-10 \mathrm{mV}(\mathrm{C}=10 \mathrm{mM})$ \\
\hline
\end{tabular}




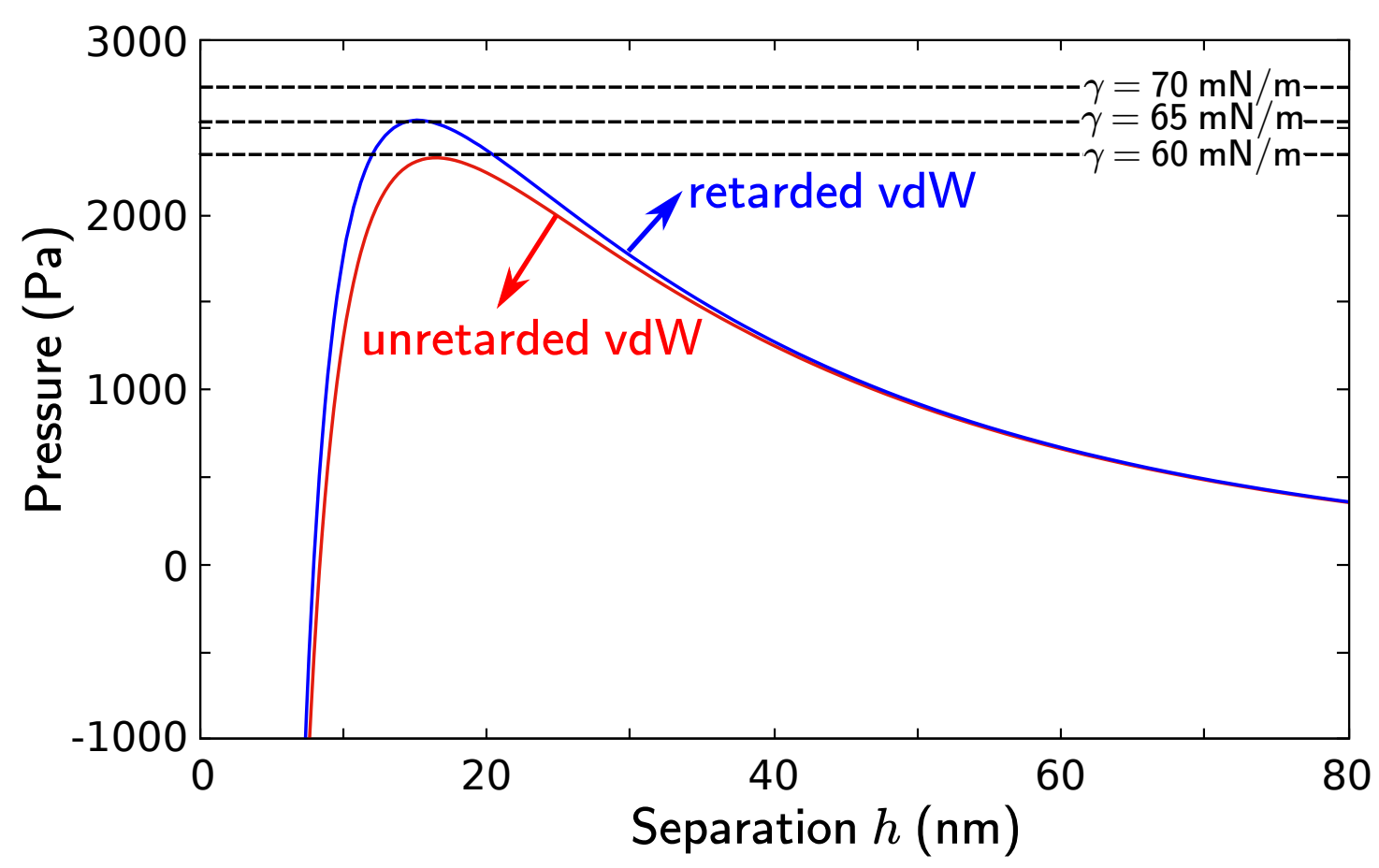

Figure S1. DLVO disjoining pressure between two air bubbles in water, with a binary electrolyte concentration of $0.1 \mathrm{mM}$ and a surface potential of $-72 \mathrm{mV}$. The red line shows the disjoining pressure calculated using a non-retarded vdW, and the blue line shows the disjoining pressure calculated using a retarded vdW. The dashed lines indicate the Laplace pressures of a drop of radius $R=51.2 \mu \mathrm{m}$ at different interfacial tensions. 

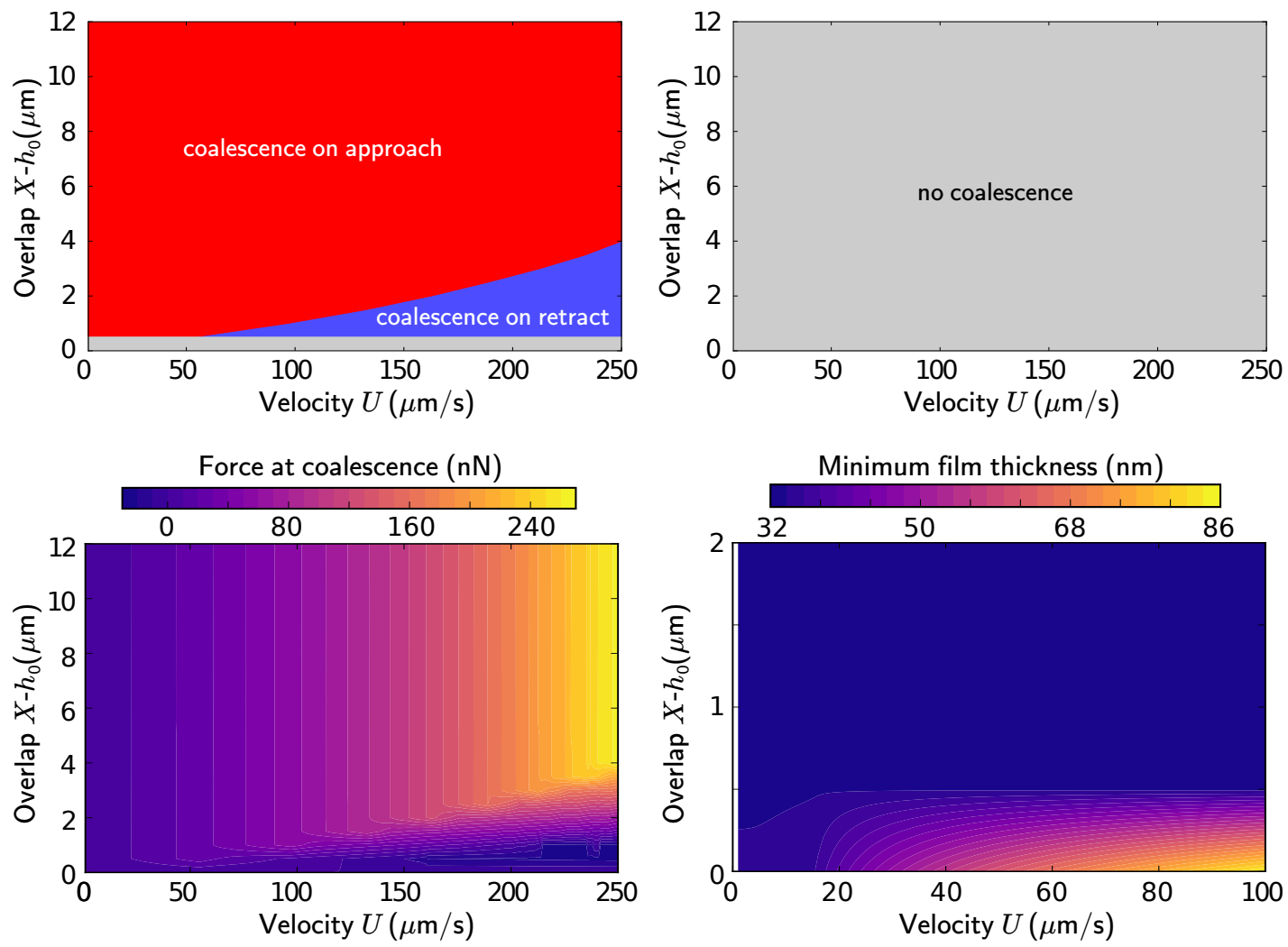

Depletion force system

Steric force system

Figure S2. a) Stability map of the interaction between two air bubbles in water under the influence of a depletion force (due to the presence of a polyelectrolyte) and an EDL force for varying drive velocities $U$ and overlaps $X-h_{0}$, and b) corresponding contour map of the force at coalescence. Consistent with Browne, et al. [1], the bubble radii $R_{1}$ and $R_{2}$ are both $50 \mu \mathrm{m}$, the surface potential is $-20 \mathrm{mV}, \sigma=$ $66 \mathrm{mN} / \mathrm{m}, T=295 \mathrm{~K}$, temperature; $B=0.2, \Delta=14.3974 \mathrm{~nm}, C_{\text {poly }}=$ $1.377649 \times 10^{25}$ monomer $/ \mathrm{m}^{3}, A=5, \xi=28.7949 \mathrm{~nm}$, and $\kappa=40.53 \mathrm{~nm}$. c) Stability map of the interaction between two decane drops in water under the influence of a steric force (due to the presence of absorbed polymers at the interface) for varying drive velocities $U$ and overlaps $X-h_{0}$, and d) corresponding contour map of the minimum film thickness for the interaction. Consistent with Manor, et al. [2], the drop radii are $R_{1}=23.6 \mu \mathrm{m}$ and $R_{2}=31 \mu \mathrm{m}$, the surface potential is -10 $\mathrm{mV}, \sigma=14.4 \mathrm{mN} / \mathrm{m}, T=295 K, \alpha=0.07, \delta=18 \mathrm{~nm}$, and $s=3.7 \mathrm{~nm}$. The red and blue regions indicate coalescence on approach and retract respectively, while the grey region represents stable interactions (where no coalescence occurs). The drops are initially separated by a distance $h_{0}$, before being driven together over a distance $X$ $=20 \mu \mathrm{m}$ with velocity $U$, and then pulled apart with velocity $-U$.

[1] C. Browne, R.F. Tabor, F. Grieser, R.R. Dagastine, Journal of Colloid and Interface Science 449 (2014) 236.

[2] O. Manor, T.T. Chau, G.W. Stevens, D.Y.C. Chan, F. Grieser, R.R. Dagastine, Langmuir 28 (2012) 4599. 


\section{University Library}

\section{- M M I E R R A A gateway to Melbourne's research publications}

Minerva Access is the Institutional Repository of The University of Melbourne

Author/s:

Berry, JD;Dagastine, RR

Title:

Mapping coalescence of micron-sized drops and bubbles

Date:

2017-02-01

Citation:

Berry, J. D. \& Dagastine, R. R. (2017). Mapping coalescence of micron-sized drops and bubbles. JOURNAL OF COLLOID AND INTERFACE SCIENCE, 487, pp.513-522. https:// doi.org/10.1016/j.jcis.2016.10.040.

Persistent Link:

http://hdl.handle.net/11343/243035 